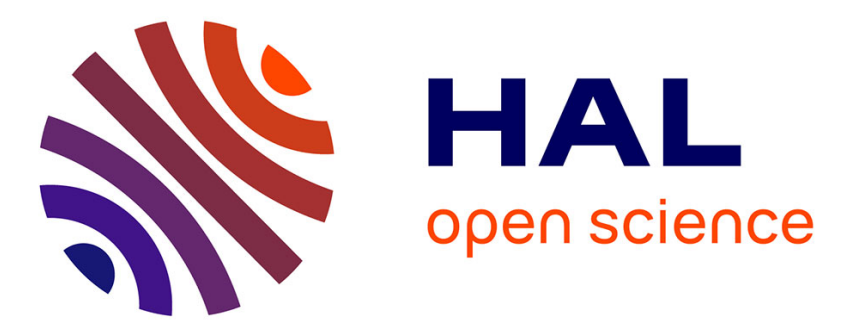

\title{
Experimental validation of theoretical methods to estimate the energy radiated by elastic waves during an impact
}

Maxime Farin, Anne Mangeney, Julien de Rosny, Renaud Toussaint, Jacques Sainte-Marie, Nikolai M. Shapiro

\section{To cite this version:}

Maxime Farin, Anne Mangeney, Julien de Rosny, Renaud Toussaint, Jacques Sainte-Marie, et al.. Experimental validation of theoretical methods to estimate the energy radiated by elastic waves during an impact. 2015. hal-01213752

\section{HAL Id: hal-01213752 \\ https://hal.science/hal-01213752}

Preprint submitted on 8 Oct 2015

HAL is a multi-disciplinary open access archive for the deposit and dissemination of scientific research documents, whether they are published or not. The documents may come from teaching and research institutions in France or abroad, or from public or private research centers.
L'archive ouverte pluridisciplinaire HAL, est destinée au dépôt et à la diffusion de documents scientifiques de niveau recherche, publiés ou non, émanant des établissements d'enseignement et de recherche français ou étrangers, des laboratoires publics ou privés. 


\title{
Experimental validation of theoretical methods to estimate the energy radiated by elastic waves during an impact
}

\author{
Maxime Farin ${ }^{\mathrm{a}, *}$, Anne Mangeney ${ }^{\mathrm{a}, 1}$, Julien de Rosny ${ }^{\mathrm{c}}$, Renaud Toussaint ${ }^{\mathrm{d}}$, \\ Jacques Sainte-Marie ${ }^{b}$, Nikolaï M. Shapiro ${ }^{a}$ \\ ${ }^{a}$ Institut de Physique du Globe de Paris, Sorbonne Paris Cité, CNRS (UMR 7154) \\ ${ }^{b}$ ANGE team, CEREMA, Inria, Lab. J.-L. Lions, CNRS \\ ${ }^{c}$ ESPCI, Institut Langevin, CNRS, 1 rue Jussieu, 75005 Paris. \\ ${ }^{d}$ Institut de Physique du Globe de Strasbourg, Université de Strasbourg / EOST, CNRS, \\ 5 rue Descartes, F-67084 Strasbourg Cedex, France.
}

\section{Abstract}

Estimating the energy lost in elastic waves during an impact is an important problem in seismology and in industry. We propose three complementary methods to estimate the elastic energy radiated by bead impacts on thin plates and thick blocks from the generated vibration. The first two methods are based on the direct wave front and are shown to be equivalent. The third method makes use of the diffuse regime. These methods are tested for laboratory experiments of impacts and are shown to give the same results, with error bars from $40 \%$ to $300 \%$ for impacts on a smooth plate and on a rough block, respectively. We show that these methods are relevant to establish the energy budget of an impact. On plates of glass and PMMA, the radiated elastic energy increases from $2 \%$ to almost $100 \%$ of the total energy lost as the bead diameter approaches the plate thickness. The rest of the

\footnotetext{
*Corresponding author

Email address: farin@ipgp.fr, +33 183957782 (Maxime Farin)
} 
lost energy is dissipated by viscoelasticity. For beads larger than the plate thickness, plastic deformation occurs and reduces the amount of energy radiated in the form of elastic waves. On a concrete block, the energy dissipation during the impact is principally inelastic because only $0.2 \%$ to $2 \%$ of the energy lost by the bead is transported by elastic waves. The radiated elastic energy estimated with the presented methods is quantitatively validated by Hertz's model of elastic impact.

Keywords: elastic waves, acoustic generation, impact source, energy budget

PACS: [2010] 46.40.-f 
Table 1: Nomenclature

$B \quad$ Bending stiffness $(\mathrm{J})$

$c_{P}, c_{S}, c_{R}$ Longitudinal, shear and Rayleigh wave speeds $\left(\mathrm{m} \mathrm{s}^{-1}\right)$

e Coefficient of restitution (-)

$e_{t o t}, e_{c}, e_{p}$ Bulk densities of total, kinetic and potential energies $\left(\mathrm{J} \mathrm{m}^{-3}\right)$

$\tilde{e}_{t o t}, \tilde{e}_{c}, \tilde{e}_{p}$ Time Fourier transform of $e_{t o t}, e_{c}, e_{p}$, respectively $\left(\mathrm{J} \mathrm{m}^{-2}\right)$

E Young's modulus $(\mathrm{Pa})$

$E_{c}, \Delta E_{c} \quad$ Energy of the impact and energy lost during the impact (J)

$E_{t o t}(t)$ Total elastic energy radiated within the structure at time $t(\mathrm{~J})$

$f \quad$ Frequency $\left(\mathrm{s}^{-1}\right)$

$\tilde{G}_{z z}^{P}, \tilde{G}_{z z}^{S}, \tilde{G}_{z z}^{R} \quad$ Vertical Green's functions associated with

compressional, shear and Rayleigh waves $\left(\mathrm{kg}^{-1} \mathrm{~s}^{2}\right)$

$h \quad$ Plate thickness $(\mathrm{m})$

$k$ Wave number $\left(\mathrm{m}^{-1}\right)$

$L, S, V$ Length $(\mathrm{m})$, surface area $\left(\mathrm{m}^{2}\right)$ and volume $\left(\mathrm{m}^{3}\right)$

$m$ Bead mass $(\mathrm{kg})$

$r, \theta, z$ Coordinates in the cylindrical reference frame $(\mathrm{m})$

$S_{i j} \quad$ Strain tensor (-)

$t$ Time (s)

$T_{i j} \quad$ Stress tensor $(\mathrm{Pa})$

$\mathbf{u}_{i} \quad$ Normalized vector of direction $i$

$u_{i}, v_{i}, a_{i}$ Surface displacement, speed and

acceleration in the direction $\overrightarrow{u_{i}}\left(\mathrm{~m} ; \mathrm{m} \mathrm{s}^{-1} ; \mathrm{m} \mathrm{s}^{-2}\right)$ 
$\tilde{U}_{i}, \tilde{V}_{i}, \tilde{A}_{i}$ Time Fourier transform of $u_{i}, v_{i}$ and $a_{i}$, respectively (m s; $\mathrm{m} ; \mathrm{m} \mathrm{s}^{-1}$ )

$v_{g}, v_{\phi} \quad$ Group and phase velocities $\left(\mathrm{m} \mathrm{s}^{-1}\right)$

$V_{z} \quad$ Speed of a bead before impact $\left(\mathrm{m} \mathrm{s}^{-1}\right)$

$W_{e l}, W_{e l}^{t h}$ Radiated energy and theoretical radiated energy $(\mathrm{J})$

$x, y, z$ Coordinates in the Cartesian reference frame (m)

$\beta, \xi$ Parameters involved in energy calculations

$\gamma$ Attenuation coefficient of energy with distance $\left(\mathrm{m}^{-1}\right)$

$\lambda, \mu$ Lamé coefficients of compression and shear (Pa)

$\nu \quad$ Poisson's coefficient (-)

$\pi_{P}, \pi_{S}, \pi_{R} \quad$ Energy partitions among $P, S V$ and Rayleigh waves (-)

$\pi_{P}^{\text {surf }}, \pi_{S}^{\text {surf }}, \pi_{R}^{\text {surf }} \quad$ Surface energy partitions among compressional, shear and Rayleigh waves (-)

$\tilde{\Pi} \quad$ Energy density flux $\left(\mathrm{J} \mathrm{m}^{-1} \mathrm{~s}\right)$

$\rho$ Density $\left(\mathrm{kg} \mathrm{m}^{3}\right)$

$\tau$ Characteristic time of energy attenuation (s)

$\chi, \eta \quad$ Viscoelastic coefficients of compression and shear (Pa s)

$\omega$ Angular frequency $\left(\mathrm{s}^{-1}\right)$ 


\section{Introduction}

The quantification of the energy emitted by a source in the form of elastic waves is a common problem in various fields such as vibroacoustics or shielding. In seismology, the problem was confronted long ago [1] and many approaches have since been developed to estimate the energy of natural sources such as earthquakes [see 2, 3, 4, 5], tremors [6], landslides and rockfalls [e.g. 7, 8, 9, 10, 11]. In the literature, the power spectral density (PSD) of the emitted signal is often measured to quantify the relative energy of different acoustic sources located at the same distance from the sensor and to compare their frequency content. For example, the temporal evolution of the PSD can provide information on river discharge and on the grain size of the bed load [e.g. 12]. The PSD can also be used to characterize crack formation in brittle [13, 14] or granular materials [see 15, for review] and other crackling or crumpling processes [e.g. 16, 17]. Finally, acoustic measurements can be useful in industry for particle sizing in powder transport and in particle streams [e.g. 18, 19]. However, the PSD does not provide an absolute estimate of the elastic energy radiated by the source because it depends on the distance of measurement.

There are three main approaches to determine the absolute radiated elastic energy from acoustic emissions. The first method consists in computing the energy flux crossing a surface surrounding the source. The integration of the energy flux over this surface gives the radiated power. This technique is applied in seismology to estimate the energy radiated in elastic waves during earthquakes [e.g. 5, 20] and rockfalls [e.g. 8, 9, 10].

The second technique to deduce the radiated elastic energy is based on 
the estimation of the time dependence of the source force. Miller and Pursey [21] and Goyder and White [22] thus estimated the power radiated in an elastic half-space and in an infinite plate, respectively, by a monochromatic harmonic force. In most cases, the force profile is generally unknown but it can be retrieved from the deconvolution of the displacement field with the Green's function tensor [3].

These two first methods can however be performed only when the emitted wave front is not mixed with its reflections off the boundaries of the elastic solid. If multiple side reflections occur, the transported energy becomes homogeneously distributed within the elastic solid and decreases exponentially with time due to viscoelastic dissipation. This situation is commonly referred to as a diffuse field in the literature [see 23, 24, 25]. A third energy estimation method, called the diffuse method hereafter, thus consists in extrapolating the radiated energy at the instant of the source from the exponential decrease of the signal coda [see e.g. 25, 26, and references therein].

The energy flux, deconvolution and diffuse field methods to estimate the energy lost in elastic waves are used separately by different communities and are based on different assumptions. The first two methods require a sufficiently large elastic solid so that the direct wave front can be clearly distinguished from its reflections off the lateral sides of the elastic solid. On the contrary, with the diffuse method, the elastic solid must be small enough so that multiple side reflections occur. To our knowledge, no study has ever compared these three methods in cases where all three can be applied.

The complex seismic signals generated by rockfalls, bed load transport in rivers and granular flows are partially composed of waves generated by the 
collisions of individual impactors (gravels, boulders,...). Therefore, if we hope to understand these signals, we must first understand the energy budget of individual impacts. The energy that is not radiated in elastic waves during an impact is lost by plastic deformation i.e., not reversible, of the impactor or of the surface [27], by local viscoelastic dissipation around the contact [28] and by conversion into other degrees of freedom of the impactor's motion, such as rotation and other displacement modes. Because of the significant differences between the conditions of each impact on the field, it is however not clear how the energy budget of the impactor depends on its size and speed.

In this paper, we propose to use the three methods introduced above to estimate the elastic energy radiated during an individual impact. Steel beads of various diameters are dropped from different heights on two glass and PMMA plates and on a concrete block and the vibration emitted by the impacts is measured with piezoelectric accelerometers. Our main objective is to quantify (i) the differences between the energy estimates and (ii) the errors made using each of the methods. Thin plates are often used in laboratory experiments because they are easier to manipulate than thick blocks. In contrast, the problem of waves generation in thick blocks is that encountered on the field. We will show that the methods to estimate the radiated elastic energy in these two geometries are different because different waves are generated. An advantage of the laboratory experiments is that the total energy lost by a rebounding bead can be easily measured from the ratio of the bead velocity after rebound over the approach velocity, i.e. the coefficient of restitution $e$ [e.g. 28]. Therefore, we can establish the energy budget of 
the impacts and observe how the percentages of energy radiated in elastic waves and dissipated by inelastic processes vary for bead impacts of different diameters and impact speeds on the thin plates and thick block investigated.

Section 2 of the paper presents the three methods to derive the energy lost in elastic waves during an impact on thin plates and thick blocks from the normal surface vibration. In section 3 , the three methods are compared for laboratory experiments of beads impacts. We also quantify the proportion of the total energy radiated in elastic waves and dissipated in inelastic processes. In section 4, we discuss the conditions of applicability of the presented methods. Finally, we evaluate the ability of the analytical model of elastic impact of Hertz [29] [see 30] to predict the radiated elastic energy and the ratio of this energy over the initial energy of the impactor when inelastic dissipation occurs.

\section{Estimation of the radiated elastic energy}

\subsection{Thin plates}

A force $\mathbf{F}(t)=-F_{z}(t) \mathbf{u}_{z}$ is applied normally at a given position $(x, y, 0)$ on the surface $(z=0)$ of a homogeneous and isotropic thin plate (Figure 1). The expression "thin plate" means that the impact duration is longer than the two-way travel time of the compressional wave in the plate thickness. The emitted elastic waves propagate radially from the impact location (direction $\mathbf{u}_{r}$, Figure 1). We consider that the principal mode excited in plates is the fundamental mode $A_{0}$ of Lamb, for which the direction of vibration is mainly normal to the plate surface (i.e. direction $\mathbf{u}_{z}$, Figure 1) [e.g. 35]. This assumption is verified experimentally in Appendix A. For all the methods 
tested below, it is therefore assumed that the vibration is only along direction $\mathbf{u}_{z}$ (Figure 1)

The mode $A_{0}$ of Lamb is highly dispersive at low frequencies, when the wavelength is much greater than the plate thickness $h$, i.e. within the limit $k h<<1$ where $k$ is the wave number. Indeed, in this regime the mode $A_{0}$ behaves as a flexural wave for which the relation between the angular frequency $\omega$ and the wave number $k$, i.e. the dispersion relation is [35]:

$$
\omega=k^{2} \sqrt{\frac{B}{\rho h}}
$$

where $\rho$ is the plate density. The bending stiffness $B$ is defined by $B=$ $h^{3} E /\left(12\left(1-\nu^{2}\right)\right)$, where $E$ and $\nu$ are the Young's modulus and Poisson's ratio of the plate material, respectively. The propagation speed of the energy, i.e. the group velocity $v_{g}=\partial \omega / \partial k$, therefore also depends on the wave number $k$ (i.e. on the angular frequency $\omega$ ):

$$
v_{g}(\omega)=2 k \sqrt{\frac{B}{\rho h}} .
$$

\subsubsection{Energy flux method}

The first method to estimate the radiated elastic energy is based on energy flux conservation on the first wave arrival. The energy density flux $\tilde{\Pi}(\omega)$ at frequency $\omega$ is by definition the bulk density of the total energy $\tilde{e}_{t o t}(\omega)=\tilde{e}_{c}(\omega)+\tilde{e}_{p}(\omega)$, integrated over plate thickness $h$, multiplied by the energy speed. But for elastic waves propagating in a homogeneous guide (for example a plate) such as the $A_{0}$ mode, the energy speed is equal to the group velocity $v_{g}(\omega)$ [35], so that:

$$
\tilde{\Pi}(\omega) \hat{=} v_{g}(\omega) \int_{-h / 2}^{h / 2} \tilde{e}_{t o t}(\omega) \mathrm{d} z
$$


Moreover, for guided waves the bulk densities of kinetic and potential energies $\tilde{e}_{c}(\omega)$ and $\tilde{e}_{p}(\omega)$ are equal [e.g. 35]:

$$
\tilde{e}_{c}(\omega)=\tilde{e}_{p}(\omega)=\frac{1}{2} \rho\left|\tilde{V}_{z}(r, \omega)\right|^{2},
$$

where $\tilde{V}_{z}(r, \omega)$ is the time Fourier transform of the surface vibration speed $v_{z}(r, t)$.

By definition, the elastic energy $W_{e l}$ radiated within the plate is given by [e.g. 35]:

$$
W_{e l} \hat{=} \int_{-\infty}^{+\infty} F_{z}\left(\mathbf{r}_{\mathbf{0}}, t\right) v_{z}\left(\mathbf{r}_{\mathbf{0}}, t\right) \mathrm{d} t
$$

where $\mathbf{r}_{\mathbf{0}}$ is the position of force application. According to Parceval's theorem, this expression is equivalent to the integral over the frequencies $\omega$ of the radiated power, which is the flux $\tilde{\Pi}(\omega)$ integrated over a line surrounding the impact:

$$
\begin{aligned}
W_{e l} & =\frac{1}{2 \pi} \int_{-\infty}^{+\infty}[\oint \tilde{\Pi}(\omega) r \mathrm{~d} \theta] \mathrm{d} \omega \\
W_{e l} & =\frac{1}{\pi} \int_{0}^{+\infty}\left[v_{g}(\omega) \iint_{S} \rho\left|\tilde{V}_{z}(r, \omega)\right|^{2} r \mathrm{~d} \theta \mathrm{d} z\right] \mathrm{d} \omega .
\end{aligned}
$$

As waves propagate radially from the source, one can integrate the surface element $r \mathrm{~d} \theta \mathrm{d} z$ over a cylinder of height equal to the plate thickness $h$ and of radius equal to the distance $r$ between the impact and the position of measurement (Figure 11). In equation (7), the distance $r$ compensates the geometrical attenuation in $1 / r^{1 / 2}$ of the vibration amplitude $\tilde{V}_{z}(r, \omega)$ of the guided wave. In addition, other dissipation is due to the intrinsic viscosity of the plate. This dissipation can be modeled by $\exp (-\gamma(\omega) r)$, where $\gamma$ is the coefficient representing the frequency-dependent attenuation of energy with 
distance $r$ in the plate (see Appendix B):

$$
W_{e l}=\int_{0}^{+\infty} 2 r h \rho v_{g}(\omega)\left|\tilde{V}_{z}(r, \omega)\right|^{2} \exp (\gamma(\omega) r) \mathrm{d} \omega .
$$

Note that if we consider a constant group velocity $v_{g}$, we obtain an expression for $W_{e l}$ similar to that used by Hibert et al. [10] to estimate the energy of surface waves generated by rockfalls in a homogeneous surface layer of depth $h$ in Dolomieu crater, Réunion Island.

\subsubsection{Deconvolution method}

As opposed to the energy flux method, here we compute the radiated elastic energy $W_{e l}$ using equation (5) from the estimation of the time dependence of the force of impact. Indeed, the energy $W_{e l}$ transferred into the plate at the point of application of a normal force $F_{z}\left(\mathbf{r}_{\mathbf{0}}, t\right)$ is the time integral of the radiated power, which is given by Goyder and White [22]:

$$
\mathbf{F}\left(\mathbf{r}_{\mathbf{0}}, t\right) \cdot \mathbf{v}\left(\mathbf{r}_{\mathbf{0}}, t\right)=\frac{F_{z}\left(\mathbf{r}_{\mathbf{0}}, t\right)^{2}}{8 \sqrt{B \rho h}}
$$

Then, according to Parceval's theorem,

$$
W_{e l}=\frac{1}{\pi} \int_{0}^{+\infty} \frac{\left|\tilde{F}_{z}(\omega)\right|^{2}}{8 \sqrt{B \rho h}} \mathrm{~d} \omega
$$

We can deduce the normal force $\tilde{F}_{z}(\omega)$ in time Fourier domain from the expression of the first arrival of the vertical vibration speed $\tilde{V}_{z}(r, \omega)$ as a function of the plate Green's function $\tilde{G}_{z z}(r, \omega)$ [3]:

$$
\tilde{V}_{z}(r, \omega)=i \omega \tilde{G}_{z z}(r, \omega) \tilde{F}_{z}(\omega)
$$

where the modulus of the plate Green's function can be approximated by, for $k r>>1$ [e.g. 36] :

$$
\left|\tilde{G}_{z z}(r, \omega)\right|=\frac{1}{8 B k^{2}} \sqrt{\frac{2}{\pi k r}}
$$


Finally, the radiated elastic energy $W_{e l}$ is given by:

$$
W_{e l}=\frac{1}{8 \pi \sqrt{B \rho h}} \int_{0}^{+\infty} \omega^{-2} \frac{\left|\tilde{V}_{z}(r, \omega)\right|^{2}}{\left|\tilde{G}_{z z}(r, \omega)\right|^{2}} \exp (\gamma(\omega) r) \mathrm{d} \omega .
$$

where $\exp (-\gamma(\omega) r)$ models the viscoelastic dissipation.

Interestingly, if we replace the Green's function $\left|\tilde{G}_{z z}(r, \omega)\right|$ by its expression [equation (12)], we retrieve the same expression of $W_{e l}$ as for the energy flux method under the condition that $\omega=k^{2} \sqrt{B / \rho h}$, which is valid for $k h<<1$. Therefore, the two methods are equivalent at low frequencies $\omega<<\sqrt{B / \rho h} / h^{2}$.

Note that the operation of dividing the amplitude of the vibration $\left|\tilde{V}_{z}(r, \omega)\right|$ by the Green's function $\left|\tilde{G}_{z z}(r, \omega)\right|$ is not trivial because the inverse Green's function diverges when $k$ (or $\omega$ ) tends towards 0 [see e.g. 31, 37]. Therefore, we cannot deconvolve the signal and estimate the energy $W_{e l}$ below a cutoff frequency. In practice, we cut all frequencies below $3 \mathrm{kHz}$ in the amplitude spectrum $\left|\tilde{V}_{z}(r, \omega)\right|$ before dividing it by the Green's function. Using a synthetic signal obtained by the convolution of the Hertz force for the elastic impact of bead diameters smaller than $20 \mathrm{~mm}$ with the Green's function in equation (12), we estimate that the energy $W_{e l}$ of the signal after the cutoff at $3 \mathrm{kHz}$ is less than $5 \%$ smaller than the exact radiated elastic energy (Figure 2).

\subsubsection{Diffuse method}

This technique is derived from classical methods used in room acoustics [see e.g. 25, and references therein]. When the emitted wave is reflected off the boundaries many times, the elastic field becomes diffuse, i.e. homogeneously distributed over the plate and equipartitioned. When the field is 
equipartitioned, the potential and kinetic energy are equal. At a given time $t$, the average over several periods (noted $\bar{x}$ ) of the total energy $E_{\text {tot }}(t)$ within the plate therefore satisfies:

$$
\overline{E_{t o t}(t)} \approx \rho h S \overline{v_{z}(t)^{2}} .
$$

where $\rho, h$ and $S$ are respectively the plate density, thickness and surface and $\overline{v_{z}(t)^{2}}$ is the average of the normal squared vibration speed $v_{z}(r, t)^{2}$ over several periods. When the field is diffuse, energy losses due to viscoelastic dissipation are proportional to the total energy within the structure:

$$
\frac{\mathrm{d} \overline{E_{t o t}(t)}}{\mathrm{d} t} \approx-\frac{\overline{E_{t o t}(t)}}{\tau},
$$

with $\tau$, the characteristic time of energy dissipation. In a narrow frequency range centered on $\omega_{0}$, this time equals $\left(\gamma\left(\omega_{0}\right) v_{g}\left(\omega_{0}\right)\right)^{-1}$ (see Appendix B). As a consequence, the energy decreases exponentially with time:

$$
\overline{E_{\text {tot }}(t)} \approx \overline{E_{t o t}\left(t_{0}\right)} \exp \left(-\frac{t-t_{0}}{\tau}\right),
$$

where $t_{0}$ is the instant of the impact. The elastic energy radiated in the plate at the instant $t_{0}$ is therefore:

$$
W_{e l}=\overline{E_{t o t}\left(t_{0}\right)} \approx \rho h S \overline{v_{z}\left(t_{0}\right)^{2}} .
$$

Knowing the instant of impact $t_{0}$ and the characteristic time $\tau$ is sufficient to determine the radiated elastic energy $W_{e l}$. Note that $\overline{v_{z}\left(t_{0}\right)^{2}}$ may fluctuate with the position of vibration measurement depending on how the assembly of proper modes of the plate are excited. Equation (17) requires that only one mode is excited within the plate because the characteristic time $\tau$ of energy 
attenuation depends on the mode. Therefore, we assume that no mode conversion occurs off the plate boundaries between the normally vibrating mode $A_{0}$ and transversal horizontal $(T H)$ or longitudinal $\left(S_{0}\right)$ modes. This hypothesis is valid provided that the plate boundaries are straight and smooth [e.g. 35].

\subsection{Thick blocks}

A force $\mathbf{F}(t)=-F_{z} \mathbf{u}_{z}$ is applied normally at a given position $(x, y, 0)$ over the surface $(z=0)$ of a homogeneous and isotropic thick block (Figure 3). The expression "thick block" means that the duration of impact is shorter than the two-way travel time of the compressional wave from the closest side of the block.

The problem of wave generation in a semi-infinite solid is commonly referred as Lamb's problem [1]. It has been treated many times for various sources below the surface [e.g. 1, 3, 38] and at the surface [e.g. 1, 21, 38, 39]. The elastic energy $W_{e l}$ initially input by a normal surface force within blocks is distributed among three different modes: compressional wave $P$, shear vertical wave $S V$ and surface Rayleigh waves. Sánchez-Sesma et al. [40] give the partitions $\pi_{P}, \pi_{S}$ and $\pi_{R}$ of energy radiated in $P, S V$ and Rayleigh waves respectively, as a function of the Poisson ratio $\nu$. For a concrete block with $\nu=0.4$, the energy partition is $\pi_{R} \approx 61 \%$ in Rayleigh waves, $\pi_{S} \approx 35 \%$ in $S V$ waves and only $\pi_{P} \approx 4 \%$ in $P$ waves.

The vibration propagating at the surface of the block contains Rayleigh waves but also compressional and shear waves as shown by the expression of the Green's function $\tilde{G}_{z z}$ owing to a normal surface force (Appendix C):

$$
\tilde{G}_{z z}=\tilde{G}_{z z}^{P}+\tilde{G}_{z z}^{S}+\tilde{G}_{z z}^{R}
$$


where $\tilde{G}_{z z}^{P}, \tilde{G}_{z z}^{S}$ and $\tilde{G}_{z z}^{R}$ are the contributions of each mode:

$$
\begin{aligned}
\tilde{G}_{z z}^{P}(r, \omega) & \approx-\frac{i}{\mu} A_{P} \frac{k_{1}}{\left(k_{1} r\right)^{2}} \exp \left(-i \omega r / c_{P}\right), \\
\tilde{G}_{z z}^{S}(r, \omega) & \approx-\frac{i}{\mu} A_{S} \frac{k_{1}}{\left(k_{1} r\right)^{2}} \exp \left(-i \omega r / c_{S}\right), \\
\tilde{G}_{z z}^{R}(r, \omega) & \approx-\frac{i}{\mu} A_{R} k_{1} \sqrt{\frac{2}{\pi k_{1} r}} \exp \left(-i\left(\omega r / c_{R}-\frac{\pi}{4}\right)\right) .
\end{aligned}
$$

In these equations, $A_{P}, A_{S}$ and $A_{R}$ are functions of Poisson's ratio $\nu$ (Appendix C), $c_{P}, c_{S}$ and $c_{R}$ are the compressional, shear and Rayleigh wave speeds, respectively, $\mu$ is the Lamé shear modulus and $k_{1}=\omega / c_{P}$ is the wave number. The expressions of these Green's functions show that the energy of compressional and shear waves at the surface decreases with frequency $f$ and distance $r$ as $(f r)^{-4}$ while the energy of Rayleigh waves varies as $f / r$ because they are guided at the surface. Therefore, the Rayleigh waves dominate the signal at high frequencies and far from the impact [1, 21].

In the following, we apply the energy flux and deconvolution methods on the Rayleigh waves to deduce the absolute radiated elastic energy $W_{e l}$. Consequently, we need to determine the percentage $\pi_{R}^{\text {surf }}(r)$ of Rayleigh waves in the energy at the position $r$ from the impact. To that end, we compute the impact force from Hertz's elastic model [e.g. 30] (Figure 4a) and convolve it with the Green's functions $\tilde{G}_{z z}^{P}, \tilde{G}_{z z}^{S}$ and $\tilde{G}_{z z}^{R}$ and the total Green's function at $r=20 \mathrm{~cm}$ on concrete (Figure $4 \mathrm{~b}$ ) to obtain the synthetic vibration acceleration $a_{z}(r, t)$ associated with each mode (Figure 4re). The compressional wave arrives clearly before the other modes. However, shear and Rayleigh waves arrive roughly at the same time and are mixed together. The total vibration acceleration $a_{z}(r, t)$ is very similar to that of the Rayleigh waves with the exception of the small wavelet corresponding to the compressional 
wave. Because shear and Rayleigh waves are out of phase, the maximum amplitude of the total vibration acceleration is $12 \%$ lower than that of the Rayleigh waves only and its squared integral is $18 \%$ lower.

The contribution of each mode $n$ to the signal energy as a function of the frequency $f$ is therefore simply $\left|\tilde{A}_{z}^{n}(r, f)\right|^{2} / \sum_{i}\left|\tilde{A}_{z}^{i}(r, f)\right|^{2}$, where $\left|\tilde{A}_{z}^{n}(r, f)\right|$ is the amplitude spectrum of the signal $a_{z}^{n}(r, t)$ associated with the $n^{\text {th }}$ mode (Figure $4 \mathrm{~d}$ ). Shear waves dominate the signal at low frequencies up to about $f=7000 \mathrm{~Hz}$, where Rayleigh waves become overriding. The percentage of compressional waves is much smaller $(<10 \%)$ and decreases with frequency. For frequencies greater than $30 \mathrm{kHz}$, the surface vibration contains only Rayleigh waves. The integration of these energy partitions over the frequencies $f$ gives the percentages of Rayleigh, compressional and shear waves at the surface (Figures 4 and 4f) ). For example, the percentages for a $5 \mathrm{~mm}$ diameter steel bead dropped from a height of $10 \mathrm{~cm}$ at $r=20 \mathrm{~cm}$ on a concrete block $(\nu=0.4)$ are respectively $\pi_{R}^{\text {surf }}=98.5 \%, \pi_{P}^{\text {surf }}=0.1 \%$ and $\pi_{S}^{\text {surf }}=1.4 \%$. Note that, at a given distance from the impact, the percentage $\pi_{R}^{\text {surf }}$ of Rayleigh waves decreases as the bead diameter $d$ increases (Figure 45) and the height of fall $H$ decreases (Figure 4 f). For example, at $r=20$ $\mathrm{cm}$, Rayleigh waves represent $99.9 \%$ of the signal for $d=1 \mathrm{~mm}$ while only about $71 \%$ for $d=20 \mathrm{~mm}$ (Figure 4e). In other words, if we assume that the signal contains only Rayleigh waves at $r=20 \mathrm{~cm}$ from the impact, the error introduced in the energy $W_{e l}$ is negligible for a bead of diameter $d=1 \mathrm{~mm}$ but is about $30 \%$ for $d=20 \mathrm{~mm}$. On the other hand, the influence of the height of fall $H$ on this percentage is negligible over the range of heights investigated here $(5 \mathrm{~cm}$ to $50 \mathrm{~cm}$, Figure $4 \mathrm{f})$. 
306

For the last method, based on the diffuse field approximation, the partitions $\pi_{R}$ and $\pi_{R}^{\text {surf }}(r)$ indicated above are no longer valid because the energy is distributed over the three directions of space $x, y$ and $z$. In this case, we use the horizontal to vertical amplitude ratio

$$
\left(\frac{\mathcal{H}}{\mathcal{V}}\right)_{\text {diffuse }}=\frac{\left|\tilde{V}_{x}(r, \omega)\right|+\left|\tilde{V}_{y}(r, \omega)\right|}{\left|\tilde{V}_{z}(r, \omega)\right|}
$$

calculated by [40] for diffuse fields, to deduce the radiated elastic energy $W_{e l}$ from the normal surface vibration speed $\tilde{V}_{z}(r, \omega)$, using the same method as for plates (see section 2.1.3).

\subsubsection{Energy flux method}

We can estimate the absolute energy radiated in elastic waves $W_{e l}$ from the energy transported by Rayleigh waves. Because Rayleigh waves propagates radially from the impact location, their energy $W_{e l}^{R}$ is calculated similarly to the radiated elastic energy in plates [equation (17)]:

$$
W_{e l}^{R}=\frac{1}{\pi} \int_{0}^{+\infty}\left[\rho v_{g} \iint_{S}\left|\tilde{V}^{R}(r, z, \omega)\right|^{2} r \mathrm{~d} \theta \mathrm{d} z\right] \mathrm{d} \omega
$$

Rayleigh waves have a elliptical motion parallel to the direction of propagation and normal to the surface, their vibration speed can therefore be written $\tilde{\mathbf{V}}^{\mathbf{R}}=\tilde{V}_{r}^{R} \mathbf{u}_{r}+\tilde{V}_{z}^{R} \mathbf{u}_{z}$ (Figure 3) [e.g. 3]. The asymptotic amplitudes far from the source of the vibration speeds $\tilde{V}_{r}^{R}$ and $\tilde{V}_{z}^{R}$ are given as a function of depth $z$ by Miller and Pursey [21]:

$$
\begin{aligned}
\left|\tilde{V}_{r}^{R}(r, z, \omega)\right| & \approx \omega \frac{\tilde{F}_{z}(\omega)}{\mu f_{0}^{\prime}\left(x_{0}\right)} \sqrt{\frac{\pi k_{1} x_{0}^{3}}{2 r}}\left(2 \sqrt{x_{0}^{2}-1} \sqrt{x_{0}^{2}-\xi^{2}} e_{\xi}-\left(2 x_{0}^{2}-\xi^{2}\right) e(\xi 4)\right. \\
\left|\tilde{V}_{z}^{R}(r, z, \omega)\right| & \approx \omega \frac{\tilde{F}_{z}(\omega)}{\mu f_{0}^{\prime}\left(x_{0}\right)} \sqrt{\frac{\pi k_{1} x_{0}\left(x_{0}^{2}-1\right)}{2 r}}\left(2 x_{0}^{2} e_{\xi}-\left(2 x_{0}^{2}-\xi^{2}\right) e_{1}\right),
\end{aligned}
$$


where $\mu$ is the Lamé shear modulus, $k_{1}=\omega / c_{P}$, with angular frequency $\omega=$ $2 \pi f$ and compressional wave speed $c_{P}, f_{0}(x)=\left(2 x^{2}-\xi^{2}\right)^{2}-4 x^{2} \sqrt{\left(x^{2}-1\right)\left(x^{2}-\xi^{2}\right)}$, $x_{0}$ is the positive root of $f_{0}$ (Figure 5) $, \xi=\sqrt{2(1-\nu) /(1-2 \nu)}, \nu$ is Poisson's ratio, $e_{\xi}=\exp \left(-k_{1} z \sqrt{x_{0}^{2}-\xi^{2}}\right)$ and $e_{1}=\exp \left(-k_{1} z \sqrt{x_{0}^{2}-1}\right)$. From these equations, we deduce that the total vibration speed $\tilde{V}^{R}$ is related to its vertical component $\tilde{V}_{z}^{R}$ by:

$$
\left|\tilde{V}^{R}(r, z, \omega)\right|^{2}=\left|\tilde{V}_{z}^{R}(r, z, \omega)\right|^{2}\left[1+\left(\frac{\mathcal{H}}{\mathcal{V}}\right)_{R}^{2}\right]
$$

with

$$
\left(\frac{\mathcal{H}}{\mathcal{V}}\right)_{R}=\frac{\left|V_{r}^{R}(r, z, \omega)\right|}{\left|V_{z}^{R}(r, z, \omega)\right|}=\frac{x_{0}}{\sqrt{x_{0}^{2}-1}} \frac{2 \sqrt{x_{0}^{2}-1} \sqrt{x_{0}^{2}-\xi^{2}} e_{\xi}-\left(2 x_{0}^{2}-\xi^{2}\right) e_{1}}{2 x_{0}^{2} e_{\xi}-\left(2 x_{0}^{2}-\xi^{2}\right) e_{1}}
$$

Equation (25) also shows that $\tilde{V}_{z}^{R}$ decreases exponentially with depth $z$ as

$$
\tilde{V}_{z}^{R}(r, z, \omega)=\tilde{V}_{z}^{R}(r, z=0, \omega) \frac{2 x_{0}^{2} e_{\xi}-\left(2 x_{0}^{2}-\xi^{2}\right) e_{1}}{\xi^{2}} .
$$

The integral over the surface $S$ surrounding the impact in equation (23) then becomes:

$$
\iint_{S}\left|\tilde{V}^{R}(r, z, \omega)\right|^{2} r \mathrm{~d} \theta \mathrm{d} z=2 \pi r \frac{\left|\tilde{V}_{z}^{R}(r, z=0, \omega)\right|^{2}}{k_{1}} A(\nu),
$$

where $A(\nu)=\int_{0}^{+\infty}\left[1+\left(\frac{\mathcal{H}}{\mathcal{V}}\right)_{R}^{2}\right]\left(2 x_{0}^{2} e_{\xi}-\left(2 x_{0}^{2}-\xi^{2}\right) e_{1}\right)^{2} / \xi^{4} \mathrm{~d}\left(k_{1} z\right)$ is a function of Poisson's ratio $\nu$ only and equal to 1.6 for our concrete block with $\nu=0.4$.

Furthermore, as discussed earlier, the squared vibration speed of Rayleigh waves $\left|\tilde{V}_{z}^{R}(r, z=0, \omega)\right|^{2}$ represents a proportion $\pi_{R}^{\text {surf }}(r)$ of the vertical squared vibration speed $\left|\tilde{V}_{z}(r, z=0, \omega)\right|^{2}$, that also includes the effects of compressional and shear waves. Thus, using equations (23), (26) and (29), we express 
the energy $W_{R}$ of Rayleigh waves as a function of the sole vertical component of the vibration speed measured at the surface of the block:

$$
W_{e l}^{R}=2 \rho r v_{g} c_{p} \pi_{R}^{\text {surf }}(r) A(\nu) \int_{0}^{+\infty}\left|\tilde{V}_{z}(r, z=0, \omega)\right|^{2} \omega^{-1} \exp (\gamma(\omega) r) \mathrm{d} \omega,
$$

where $\exp (\gamma(\omega) r)$ counterbalances the viscoelastic dissipation of energy. In practice, we cut the frequencies below $3 \mathrm{kHz}$ in the amplitude spectrum $\left|\tilde{V}_{z}(r, z=0, \omega)\right|$ to avoid the divergence of the term within the integral as $\omega$ tends towards 0 (see section 2.1.2).

Finally, the energy $W_{e l}^{R}$ of Rayleigh waves represents only a percentage $\pi_{R}$ of the total elastic energy $W_{e l}$ radiated within the block, thus:

$$
W_{e l}=\frac{W_{e l}^{R}}{\pi_{R}}=2 \rho r v_{g} c_{p} \frac{\pi_{R}^{\operatorname{surf}}(r)}{\pi_{R}} A(\nu) \int_{0}^{+\infty}\left|\tilde{V}_{z}(r, z=0, \omega)\right|^{2} \omega^{-1} \exp (\gamma(\omega) r) \mathrm{d} \omega .
$$

\subsubsection{Deconvolution method}

Miller and Pursey [21] deduced an analytical expression for the radiated elastic energy $W_{e l}$ from the surface deformation created by the action of a point force $\tilde{F}(\omega)$ (in the time Fourier domain) on the surface of a semi-infinite solid, using equation (5):

$$
W_{e l}=\frac{\xi^{4} \beta}{2 \pi^{2} \rho c_{p}^{3}} \int_{0}^{+\infty} \omega^{2}|\tilde{F}(\omega)|^{2} \mathrm{~d} \omega,
$$

where $\beta$ is the imaginary part of

$$
\int_{0}^{X} \frac{x \sqrt{x^{2}-1}}{f_{0}(x)} \mathrm{d} x
$$

with $f_{0}(x)=\left(2 x^{2}-\xi^{2}\right)^{2}-4 x^{2} \sqrt{\left(x^{2}-1\right)\left(x^{2}-\xi^{2}\right)}, \xi=\sqrt{2(1-\nu) /(1-2 \nu)}$ and $X$ a number greater than the real root $x_{0}$ of $f_{0}$. The coefficient $\beta$ depends 
only on the Poisson ratio $\nu$ (see Appendix D for details on the calculation of $\beta)$.

In our case, the impact force $\tilde{F}(\omega)$ is vertical and can be obtained from the normal surface vibration speed $\tilde{V}_{z}^{R}(r, z=0, \omega)$ using equation (11) with the Green's function of Rayleigh waves [equation (21)]. Therefore, the radiated elastic energy $W_{e l}$ is given by:

$$
W_{e l}=\frac{\xi^{4} \beta \pi_{R}^{\mathrm{surf}}(r)}{2 \pi^{2} \rho c_{p}^{3}} \int_{0}^{+\infty} \frac{\left|\tilde{V}_{z}^{R}(r, z=0, \omega)\right|^{2}}{\left|\tilde{G}_{z z}^{R}(r, \omega)\right|^{2}} \exp (\gamma(\omega) r) \mathrm{d} \omega
$$

To compute the radiated elastic energy, we perform the same operation as in section 2.1 .2 because the inverse Green's function $1 / \tilde{G}_{z z}$ also diverges as $\omega$ tends toward 0 .

If we replace $\left|\tilde{G}_{z z}^{R}(r, \omega)\right|$ by its expression in equation (21), we obtain:

$$
W_{e l}=2 \rho r v_{g} c_{p} \pi_{R}^{\text {surf }}(r) \frac{\beta x_{0}}{8 \pi A_{R}^{2}} \int_{0}^{+\infty}\left|\tilde{V}_{z}(r, z=0, \omega)\right|^{2} \omega^{-1} \exp (\gamma(\omega) r) \mathrm{d} \omega
$$

Note that the energy $W_{e l}$ calculated with the energy flux method [equation (31)] and the energy calculated from the impact force [equation (35)] are proportional to the same integral. The discrepancy between the energies computed with the two methods can be estimated by the ratio of the coefficients in front of the integral in equations (31) and (35), i.e. $\beta x_{0} \pi_{R} / 8 \pi A_{R}^{2} A(\nu)$, which equals $1 \pm 10^{-4}$ regardless of Poisson's ratio $\nu$. The two methods are therefore equivalent.

\subsubsection{Diffuse method}

After many reflections of the wave front off the block boundaries, we assume that the energy within the block is distributed along the three directions of space, i.e. that the field is diffuse [e.g. 25]. The ratio of horizontal 
to vertical amplitude at the surface of a semi-infinite medium under a diffuse field approximation is given by Sánchez-Sesma et al. [40] for a normal loading force as a function of the Poisson ratio $\nu:\left(\frac{\mathcal{H}}{\mathcal{V}}\right)_{\text {diffuse }} \approx 1.245+0.348 \nu$. For our concrete block $(\nu=0.4),\left(\frac{\mathcal{H}}{\mathcal{V}}\right)_{\text {diffuse }} \approx 1.38$. From the hypothesis of energy equipartition, we obtain an expression for the radiated elastic energy $W_{e l}$ that is similar to that previously demonstrated for plates [equation (17)]:

$$
W \approx\left(1+\left(\frac{\mathcal{H}}{\mathcal{V}}\right)_{\text {diffuse }}^{2} \rho V \overline{v_{z}\left(t_{0}\right)^{2}}\right.
$$

where $V$ is the block volume. In the case of blocks, the factor $1+\left(\frac{\mathcal{H}}{\mathcal{V}}\right)_{\text {diffuse }}^{2}$ compensates the energy distribution over the three directions of space.

\section{Experimental test}

\subsection{Setup}

We conduct impact experiments on two thin plates and a thick block to test the three methods presented in section 2, Piezoelectric charge shock accelerometers (type 8309, Brüel \& Kjaer) record the normal acceleration generated by impacts at various positions. The surface vibration is digitalized with an acquisition rate of $0.3 \mathrm{MHz}$. The accelerometers have a rather flat response over a wide range of frequencies ( $1 \mathrm{~Hz}$ to $54 \mathrm{kHz}$ ). Note that only one accelerometer is necessary to measure the radiated elastic energy regardless of the method used because the radiated wave field is isotropic. Nevertheless, several sensors are placed at different distances from the impact to measure wave dispersion Appendix A and energy attenuation, i.e. the coefficients $\gamma$ and $\tau$ Appendix B and Table 2). 
The impactors are spherical steel beads of density $7800 \mathrm{~kg} \mathrm{~m}^{-3}$ and diameter ranging from $1 \mathrm{~mm}$ to $20 \mathrm{~mm}$. The beads are dropped from various heights from $2 \mathrm{~cm}$ to $25 \mathrm{~cm}$, without initial velocity and rotation, on a circular glass plate with a radius of $40 \mathrm{~cm}$ and thickness of $1 \mathrm{~cm}$, on a $1.2 \times 1$ $\mathrm{m}^{2}$ PMMA plate with a thickness of $1 \mathrm{~cm}$ and on a $3 \times 1.5 \times 0.6 \mathrm{~m}^{3}$ concrete block. The properties of these structures are presented in Table 2 .

\subsection{Description of the measured signals}

The two plates and the block were selected to check as comprehensively as possible the assumptions made in the previous section to calculate the radiated elastic energy. On the one hand, after each bead impact on the glass plate and on the concrete block, the accelerometers record a long coda owing to the multiple side reflections off the lateral sides of the structure (Figure $6 \mathrm{a}$ and $7 \mathrm{a}$ ). In these two structures, there are enough reflections for a diffuse field to be set up and we can apply the diffuse method to estimate the radiated elastic energy. However, it is not possible to use this method on the PMMA plate because side reflections are too attenuated (Figure 8 8 a). After about 30 side reflections in the glass plate and 10 in the concrete block, the averaged squared vibration amplitude $\left|\overline{a_{z}(r, t)}\right|^{2}$ decreases exponentially with time, until it reaches the noise level (Figures $6 \mathrm{~b}$ and $7 \mathrm{~b}$ ). We can thus estimate the characteristic time $\tau$ of energy attenuation in these structures (see Appendix B and Table 2).

On the other hand, the two plates and the block are sufficiently large to record a majority of the first arrival of the emitted vibration before the return of the first side reflection (Figures $6 c, 7 \mathrm{k}$ and $8 \mathrm{a}$ ). We can therefore apply the methods based on the first arrival i.e., the energy flux and deconvolution 
methods, to determine the elastic energy radiated by the impacts on each investigated structure.

The time Fourier transform of the first arrival gives the amplitude spectrum $\left|\tilde{A}_{z}(r, f)\right|$ (Figures 6 $\mathrm{d}, 7 \mathrm{~d}$ and $8 \mathrm{r}$ ). Impacts of beads excite a wide frequency range up to about $80 \mathrm{kHz}$ and are characterized by an energy peak with a central frequency between $2 \mathrm{kHz}$ and $40 \mathrm{kHz}$ (Figure 9). The duration of impact increases with the bead diameter and consequently the peak frequency of the generated vibration decreases. Interestingly, for impacts of beads of diameter smaller than $5 \mathrm{~mm}$ on the glass plate, the peak frequency is constant and equals $34 \mathrm{kHz}$. This is discussed in section 4.2

\subsection{Radiated elastic energy}

For experiments of bead impacts on the glass and PMMA plates, the energy flux and deconvolution methods give almost identical results (Figure 10a and 10c). The energy obtained with deconvolution is $2 \%$ greater than that obtained with the energy flux method on the glass plate and $5 \%$ greater on PMMA. On the glass plate, we also observe a fair agreement between the energy estimated using the energy flux method and the diffuse method (Figure $10 \mathrm{~b}$ ). The lower signal to noise ratio for small beads (i.e. for $W_{e l}<10^{-7}$ $\mathrm{J}$, Figure [10b) leads to an error of $+20 \%$ on the radiated elastic energy $W_{e l}$ with the diffuse method with respect to the energy flux method. However, the discrepancy between the methods is lower than the uncertainties on the energy $W_{e l}$ ( \pm 1 standard deviation). The error is about $\pm 37 \%$ with the energy flux method, $\pm 36 \%$ with the deconvolution method and $\pm 53 \%$ with the diffuse method. The error is greater $( \pm 60 \%)$ for beads smaller than $2 \mathrm{~mm}$ (i.e. for $W_{e l}<10^{-7} \mathrm{~J}$, Figure 10) because of the lower signal to noise ratio. 
For impacts on the concrete block, the radiated elastic energy $W_{e l}$ obtained with the deconvolution method is equal to that computed with the energy flux method, as discussed in section 2.2.2 (Figure 11a). The energy estimation error with these two methods is that of the integral $\int_{0}^{+\infty} \mid \tilde{V}_{z}(r, z=$ $0, \omega)\left.\right|^{2} \omega^{-1} \exp (\gamma(\omega) r) \mathrm{d} \omega$ in equations (31) and (35) and is about $\pm 75 \%$. We cannot use the diffuse method for beads smaller than $2 \mathrm{~mm}$ in diameter because not enough side reflections can be recorded. For larger beads, the energy measured with the diffuse method is between 0.3 to 3 times that obtained with the other methods (Figure 11b). Error bars with the diffuse method are between $\pm 70 \%$ and $\pm 300 \%$ and are of the same order of magnitude as the difference between the methods.

Let us discuss the possible source of errors in our experiments. For the energy flux and deconvolution methods, the error bars are greater on the block $(\approx 75 \%)$ than on the plates $(\approx 36 \%)$. This is probably because we can less clearly identify the first emitted wave train from the side reflections in the concrete block than in the plates (Figures 6k, 7k and 8b). Moreover, the rough surface of the concrete block is a likely cause for greater scattering of the results than on the smooth glass and PMMA plates, in particular for beads of diameter $d<3 \mathrm{~mm}$ for which the depth of penetration into the concrete is of the same order of magnitude as the surface roughness. The diffuse method is based on statistical assumptions that induce additional errors. First, the diffuse regime is reached after at least 30 side reflections in the glass plate and 10 in the concrete block. Consequently, if damping is important, as it is the case in concrete, the diffuse field is not completely set, the exponential decay of the energy is not clear and the characteristic 
time $\tau$ of energy dissipation is not well estimated (Figure $7 \mathrm{~b}$ ). The error on $\tau$ therefore leads to either overestimate or underestimate the radiated elastic energy. Secondly, an exponential decay of the energy assumes that the energy dissipation is frequency independent, which is not completely the case here (Table 2).

\subsection{Elastic transfer efficiency}

We measure the total energy $\Delta E_{c}$ lost by the beads from their vertical coefficient of restitution $e$ [e.g. 28, 41]. The proportion of energy radiated in elastic waves $W_{e l}$ with respect to the lost energy $\Delta E_{c}$, i.e. the elastic transfer efficiency, increases with bead diameter up to $d=5 \mathrm{~mm}$ and decreases for $d \geq 10 \mathrm{~mm}$ (Figure 12a). The ratio $W_{e l} / \Delta E_{c}$ does not depend on the fall height $H$ for impacts on the PMMA plate and concrete block (Figure 12b). On the glass plate, for bead diameters $d$ between $2 \mathrm{~mm}$ and $5 \mathrm{~mm}$ and fall heights $H>5 \mathrm{~cm}$, the radiated elastic energy $W_{e l}$ is greater than the lost energy $\Delta E_{c}$, which is impossible. We will explain this discrepancy in the discussion section. More energy is converted into elastic waves for impacts on the glass plate and on the PMMA plate than on the concrete block. Indeed, the ratio $W_{e l} / \Delta E_{c}$ is never greater than $2 \%$ on the concrete block while on the PMMA plate, almost all the lost energy is radiated elastically for bead diameters $d \geq 5 \mathrm{~mm}$ (Figure $12 \mathrm{a}$ ), regardless of the fall height $H$ (Figure $12 \mathrm{~b})$. 


\section{Discussion}

\subsection{Comparison between the different methods}

It is valid to use the energy flux and deconvolution methods when the first wave arrival can be discerned from side reflections or when the side reflections are very attenuated. The diffuse method is applicable provided that enough side reflections occur to equipartition the energy. The diffuse method therefore becomes very efficient in a small structure. Another advantage of the diffuse method is that there is no assumption on the direction of the impact force.

The three methods can be used with only one sensor to measure the radiated elastic energy but the precision of the energy estimation can be enhanced when several sensors are used. For the direct wave methods, the use of several sensors can take into account an anisotropic emission. For the diffuse method, it can compensate for a not completely equipartitioned field because we estimate the averaged value of the energy over the surface of the structure.

\subsection{Comparison with Hertz's model of elastic impact}

Impacts of spherical beads on a plane surface are often compared with Hertz's [29] theory of elastic impact [e.g. 19, 30, 31, 28, 32, 33]. For example, using equation (5) with an expression of the impact force $F_{z}\left(\mathbf{r}_{\mathbf{0}}, t\right)$ based on Hertz's theory, Hunter [32] and Reed [33] estimated the theoretical value $W_{e l}^{t h}$ of the elastic energy emitted by beads impacting thick elastic blocks. However, their approach has never been extended to the case of impacts on thin plates. Moreover, if inelastic energy dissipation occurs during the impact, 
the amplitude of the impact force is expected to decrease with respect to the elastic case [30, 28, 34] and Hertz's model may overestimate the radiated elastic energy.

To interpret our results, we compare the measured signals and amplitude spectra with synthetic signals obtained by convolution of the Green's function [equations (12) and (18)] with Hertz's force of elastic impact (Figures [6, 66 d, 75, 77d, $8 \mathrm{~b}$ and $8 \mathrm{c})$. Moreover, we also compare the measured radiated elastic energy $W_{e l}$ with the energy $W_{e l}^{t h}$ of the synthetic signal (Figure 13).

A good agreement with elastic theory is observed for the PMMA plate in terms of amplitude and frequencies (Figures $8 \mathrm{~b}$ and $8 \mathrm{k}$ ). The measured radiated energy $W_{e l}$ in PMMA is generally of the same order of magnitude but smaller than the theoretical one $W_{e l}^{t h}$ by up to a factor of 3 (Figures 13a and $13 \mathrm{~b}$ ). We used a laser Doppler vibrometer to measure the exact vibration displacement of the glass plate surface after a bead impact (Figure 14). This reveals that the system constituted by the accelerometer and the glass plate shows a resonance frequency around $38 \mathrm{kHz}$. As a consequence, the accelerometer records a greater amplitude than that of the generated vibration at frequencies close to $38 \mathrm{kHz}$ (Figure 14). This is clearly visible both on the temporal signal and amplitude spectrum when we compare them with their synthetic counterparts (Figures [6 and 6 $\mathrm{d}$ ). Indeed, the measured signal lasts much longer than the synthetic signal (Figure [6c) and the measured spectrum has a higher amplitude than the synthetic spectrum around the resonance frequency (Figure 6 $6 \mathrm{~d}$ ). Because of the resonance, the measured radiated elastic energy $W_{e l}$ is up to 4 times greater than $W_{e l}^{t h}$ for impacts of beads of diameter $d<10 \mathrm{~mm}$ on the glass plate, regardless of the fall height 
$H$ (Figures 13a and 13b). More importantly, $W_{e l}$ is even greater than the lost energy $\Delta E_{c}$ (Figures 12 and 12b), which is impossible owing to energy conservation. This resonance seems excited by impacts of beads of diameter $d \leq 5 \mathrm{~mm}$ because the peak frequency of the amplitude spectrum generated by the impacts of these beads is constant and equals $34 \mathrm{kHz}$ (Figure 9), while it should increase for decreasing bead diameter $d$ [31]. The origin of this resonance is still under study.

It is not clear whether the resonance is also observed for impacts on the concrete block because the synthetic signal is very different from the measured signal (Figures $7 \mathrm{k}$ and $7 \mathrm{~d}$ ). For example, we can discern the compressional wave and the Rayleigh wave in the synthetic signal but not in the measured signal (Figure 7k). That said, on concrete, the peak frequency of the amplitude spectrum decreases for increasing bead diameter $d$, which does not suggest resonance (Figure 91). The measured signal on concrete has smaller frequencies than the synthetic signal, probably because the duration of the impact of steel beads on this block is longer than that predicted by Hertz (Figures $7 \mathrm{k}$ and $7 \mathrm{~d}$ ). On the concrete block, the measured radiated energy $W_{e l}$ is smaller than the theoretical energy $W_{e l}^{t h}$ by up to a factor of 7 for bead diameters $d<5 \mathrm{~mm}$ and $d>10 \mathrm{~mm}$ (Figures 13a and 13 b).

For impacts on the thin plates, the variation of the energy ratio $W_{e l} / E_{c}$ with diameter $d$ is well reproduced by Hertz's theory up to $d=10 \mathrm{~mm}$, but the agreement is not quantitatively good on the glass plate, probably due to the resonance (Figures 13k and 13 d). For larger beads, however, Hertz's theory leads to values of the radiated elastic energy $W_{e l}^{t h}$ greater than the impact energy $E_{c}$, which is impossible (Figure 13c). On the concrete 
block, Hertz's model fails to reproduce the variation of the ratio $W_{e l} / E_{c}$ with bead diameter $d$ (Figure 13 c). Indeed, for an elastic impact, the ratio $W_{e l}^{t h} / E_{c}$ is independent of the bead diameter $d$ while the measured ratio $W_{e l} / E_{c}$ first increases, reaches a maximum for $d=5 \mathrm{~mm}$ and then decreases (Figure 13k). Similarly, the measured ratio $W_{e l} / E_{c}$ is roughly independent of the fall height $H$ while theory predicts it should increase (Figure 13 $\mathrm{d}$ ). The average measured ratio $W_{e l} / E_{c}$ on the block is between $0.1 \%$ and $2 \%$, which is in agreement with previous bead-drop experiments on thick blocks [32, 33, 34]. This is however several orders of magnitude higher than the ratios $W_{e l} / E_{c}=10^{-5}$ to $10^{-3}$ measured for rockfalls in the field, for which plastic deformation is much more important [9, 10].

To sum up, it is valid to use Hertz's force of elastic impact to qualitatively predict the variation of the radiated elastic energy $W_{e l}$ with bead diameter $d$ and fall height $H$ on a smooth plate when the bead diameter $d$ is smaller than the plate thickness $h$. However, the small ratio of $W_{e l}$ to the lost energy $\Delta E_{c}$ for beads of diameter $d<3 \mathrm{~mm}$ and $d>10 \mathrm{~mm}$ suggests that our experiments involve a range of bead diameters and impact speeds in which viscoelastic and plastic dissipation may occur (Figure 12a). Hertz's model does not take into account inelastic dissipation during impact, which can reduce the amplitude of the impact force and thereby decrease the amount of energy radiated by elastic waves [see 30]. The difference observed between the measured radiated elastic energy $W_{e l}$ and that predicted by Hertz's model $W_{e l}^{t h}$ can therefore be partly explained by the presence of inelastic dissipation. A more complex model is therefore needed to account for these energy losses, as discussed in the next paragraph. 


\subsection{Inelastic energy dissipation}

For a viscoelastic impact, Ramírez et al. [42] showed that the coefficient of restitution $e$ decreases with the impact speed $V_{z}$ as $1-c V_{z}^{1 / 5}$ where $c$ is a constant depending on bead diameter. This scaling law agrees well with our experimental results on the glass and PMMA plates but not with those on the concrete block (Figure 15). Some energy may therefore be dissipated viscoelastically on plates. Although not explicitly indicated by the authors, the model of Ramírez et al. [42] shows that the energy lost by viscoelastic dissipation is greater for small beads. This is in agreement with our data because the discrepancy between the measured and the theoretical energy is larger as the bead diameter $d$ decreases (Figure 12a). Additional energy losses may also occur for the smallest beads investigated $(d<3 \mathrm{~mm})$ due to surface imperfections and adhesion [31]. These effects are even greater on the concrete block with its surface roughness of $\approx 0.5-1 \mathrm{~mm}$. Therefore, the energy that is not radiated in elastic waves for beads of diameter $d<5 \mathrm{~mm}$ is likely dissipated in viscoelasticity as well as in adhesion and rotational and translational modes. On the PMMA plate, this inelastic dissipation represents from $99 \%$ to $10 \%$ of the lost energy with increasing diameter $d$ from $1 \mathrm{~mm}$ to $4 \mathrm{~mm}$ (Figure 12 $\mathrm{a}$ ). On the concrete block, this represents almost all the lost energy because the percentage of lost energy radiated in elastic waves is very small (0.1\%-2\%) (Figure $12 \mathrm{a}$ ).

The minimum impact speed necessary to deform a structure plastically is very low ( $\simeq 0.1 \mathrm{~m} \mathrm{~s}^{-1}$ for steel impacting steel [30]) and this velocity is clearly exceeded in all our experiments. However, the minimum impact speed to cause fully plastic deformation is much higher and such impacts 
are characterized by a coefficient of restitution $e$ that decreases with impact speed as $V_{z}^{-1 / 4}$ [30]. Our data do not fit this scaling law, even for the largest beads investigated (Figure 15). The impacts in our experiments are therefore elastic-plastic but not fully plastic. Plastic deformation is more likely to occur for the largest beads because higher stresses are developed during the impact. As a matter of fact, plastic deformation is evidenced on glass and concrete, but not on PMMA, by the presence of small indentations on the surface after impacts of beads larger than $10 \mathrm{~mm}$. As a consequence, the elastic transfer efficiency decreases for beads of diameter $d>5 \mathrm{~mm}$ (Figure [12 ). For a given bead diameter $d>10 \mathrm{~mm}$, the impact seems more elastic on PMMA than on glass or on concrete because the ratio $W_{e l} / \Delta E_{c}$ decreases less on PMMA than on the other structures (Figure 12a). As suggested by McLaskey and Glaser [31], PMMA is a more compliant material than glass and concrete and thereby the impacts lasts longer and over a larger area of contact, reducing the maximum stresses applied on the surface. On the plates, we estimate that the plastic deformation represents up to $20 \%$ of the lost energy for $d=20 \mathrm{~mm}$ (Figure 12a). This is however not quantifiable on the concrete block because the surface roughness may contribute to a high proportion of the energy losses.

Finally, note that even when inelastic dissipation occurs, the three methods of energy calculation compared in this paper give very similar results (Figures 10 and 11). However, plastic deformation (or surface roughness) may generate an impact force with a greater tangential component, as suggested by Buttle and Scruby [18]. This can therefore affect our estimation of the radiated elastic energy because we make the assumption that the impact 
637 force is normal to the surface. For example, Sánchez-Sesma et al. [40] showed ${ }_{638}$ that the stronger the tangential force is on the surface of a semi-infinite block, 639 the smaller the generated vertical displacement is with respect to the radial 640 displacement. 


\section{Conclusions}

We presented and validated experimentally three methods to estimate the elastic energy radiated by an impact on a thin plate and a thick block from the measurement of the surface normal vibration at a single location. The energy flux method and deconvolution methods are based on the direct wave between the impact and are shown to give the same results for both plates and blocks. The diffuse method makes use of the diffuse coda during which multiple reflections occur off the structure's borders. This last method slightly overestimates the radiated elastic energy with respect to the other methods on plates $(+5-20 \%)$, but gives results of the same order of magnitude (i.e. within a factor of 3 ) as the other methods when applied to blocks. The differences between the estimates are however less than the uncertainty of each method, with standard deviations between $40 \%$ and $70 \%$ for the energy flux and deconvolution methods and between $50 \%$ and $300 \%$ for the diffuse method.

The presented methods have the major advantage of estimating the radiated elastic energy independently with respect to the other energy dissipation processes, without knowledge of the impact force. This allowed us to establish an energy budget for the impacts:

- On thin plates, the percentage of energy lost in elastic waves increases with the bead diameter. This percentage is less than $2 \%$ of the total energy lost when the bead diameter is smaller than $10 \%$ of the plate thickness. The rest of the energy lost by the bead is likely dissipated by viscoelasticity. On the other hand, almost all the lost energy is radiated in elastic waves for bead diameters greater than the plate thickness and 
the rest is lost in plastic deformation (up to $20 \%$ in our experiments).

- On rough thick blocks, the radiated elastic energy represents only between $0.2 \%$ to $2 \%$ of the lost energy, regardless of the bead diameter and fall height. Inelastic dissipation (i.e. viscoelastic, plastic, rotational,...) is therefore the major energy consumption process.

The elastic impact model of Hertz well reproduces the measured radiated elastic energy on thin plates for bead diameters smaller than the plate thickness, but overestimates the energy for larger beads. On thick blocks, the model gives quantitatively good results but overestimates the radiated elastic energy by a factor of 2 to 10 when inelastic dissipation occurs.

Further work is required to investigate how surface roughness affects the amount of energy radiated in elastic waves and dissipated by inelastic processes during an impact. For example, it would be interesting to establish the energy budget of beads impacts on thick blocks with a surface as smooth as that of the thin plates.

\section{Acknowledgements}

We thank V. Farra, J.P. Montagner, E. Stutzmann, R. Madariaga, E. Falcon, D. Royer, A. Schubnel, T. Reuschl and L. Jouniaux for helpful discussions. We are indebted to J. Martin for his useful pieces of advice in signal processing and to A. Steyer for technical help. We thank the journal editor Ines Lopez Arteaga and two anonymous reviewers for their helpful comments to increases the clarity of our manuscript. This work was supported by the european project ERC SLIDEQUAKES, the Agence Nationale de la 
${ }_{689}$ Recherche ANR LANDQUAKES project, the REALISE project and the ITN ${ }_{690}$ FLOWTRANS project. 


\section{Appendix A. Experimental determination of the relations of dis- persion}

In this section, we detail how to determine the relations of dispersion of the structures used for impacts experiments. In order to observe wave dispersion, we measure the emitted wave front at several distances $r$ from a given bead impact (e.g. for PMMA, Figure 16a). The double Fourier transform in time and space of the vibration acceleration $a_{z}(r, t)$ allows to deduce the relation between the angular frequency $\omega$ and the wave number $k$, i.e. the dispersion relation (Figures 16b and 16k).

As expected, for the plates of PMMA and glass the dispersion relation corresponds exactly to that of the fundamental mode $A_{0}$ of Lamb (Figures 16k and 17a). At low frequencies, i.e. for $k h<1$, the dispersion relation can be approximated by $\omega \approx 5.5 k^{2}$ in PMMA and $\omega \approx 13.8 k^{2}$ in glass, thus satisfying equation (11) with a bending stiffness $B=357 \mathrm{~J}$ and $B=4760$ $\mathrm{J}$, respectively. On the other hand, the mode $A_{0}$ is not dispersive at higher frequencies, for $k h>1$. Indeed, the relation between the frequency and the wave number becomes roughly linear and the group velocity $v_{g}=\partial \omega / \partial k$ tends towards the Rayleigh wave velocity that is $\approx 1400 \mathrm{~m} \mathrm{~s}^{-1}$ for PMMA and $\approx 3100 \mathrm{~m} \mathrm{~s}^{-1}$ for glass [35].

For the glass and PMMA plates, we estimate the energy associated with the longitudinal $S_{0}$ mode with an accelerometer on the plate border. In both plates, the energy of this mode is about $0.2 \%$ of that of the vertical $A_{0}$ mode and is consequently negligible. The plates vibration is therefore mostly normal to the surface. The lowest secondary mode in plates is the mode $A_{1}$ that has a cutoff frequency equal to $c_{S} / 4 h \approx 82 \mathrm{kHz}$ in glass and 
716

$22 \mathrm{kHz}$ in PMMA, where $c_{S}$ is the shear wave speed. The accelerometers record frequencies up to $80 \mathrm{kHz}$, therefore we do not measure modes higher than the $A_{0}$ mode in glass. In PMMA, however, the mode $A_{1}$ may be present but its amplitude is too low to be detected in the dispersion curve $\omega=f(k)$ (Figure 16k).

For the concrete block, the relation between the angular frequency $\omega$ and the wave number $k$ is roughly linear with a slope of $1530 \mathrm{~m} \mathrm{~s}^{-1}$ that corresponds to both the phase $v_{\phi}$ and group $v_{g}$ velocities (Figure 17b). 


\section{Appendix B. Energy dissipation model in a viscoelastic solid}

In this Appendix, we show that the viscous dissipation of energy with distance $r$ in a Kelvin-Voigt viscoelastic solid can be modeled by a factor $\exp (-\gamma r)$ where $1 / \gamma$ is a characteristic length of energy dissipation that depends on frequency. To that end, we have to demonstrate the equation of energy conservation in such a solid. We start from the equation of momentum conservation in the solid, stating that:

$$
\rho \frac{\partial^{2} u_{i}}{\partial t^{2}}=\frac{\partial T_{i j}}{\partial x_{j}},
$$

where $u_{i}$ is the wave displacement and $T_{i j}$ is the stress tensor. The summation on repeated indices is implicit. In a homogeneous and isotropic viscoelastic solid modeled by Kelvin-Voigt model, Hooke's law is [35]:

$$
T_{i j}=T_{i j}{ }^{e l}+T_{i j}{ }^{i n e l}
$$

with

$$
T_{i j}^{e l}=\lambda \delta_{i j} S+2 \mu S_{i j}
$$

and

$$
T_{i j}^{i n e l}=\chi \delta_{i j} \frac{\partial S}{\partial t}+2 \eta \frac{\partial S_{i j}}{\partial t}
$$

where $S_{i j}=\frac{1}{2}\left(\frac{\partial u_{i}}{\partial x_{j}}+\frac{\partial u_{j}}{\partial x_{i}}\right)$ is the strain tensor and $S=\partial u_{j} / \partial x_{j}$. The constants $\lambda, \mu$ and $\chi, \eta$ are the elastic and viscous coefficients associated to compression and shear, respectively. Note that these coefficients generally depend on frequency $f$.

Multiplying equation (B.1) by $\frac{\partial u_{i}}{\partial t}$, we obtain:

$$
\frac{\partial e_{c}}{\partial t}=\frac{\partial T_{i j}^{e l}}{\partial x_{j}} \frac{\partial u_{i}}{\partial t}+\frac{\partial T_{i j}^{i n e l}}{\partial x_{j}} \frac{\partial u_{i}}{\partial t},
$$


where $e_{c}$ is the bulk density of kinetic energy.

We can develop the second term of equation (B.5) noting that:

$$
\frac{\partial T_{i j}{ }^{e l}}{\partial x_{j}} \frac{\partial u_{i}}{\partial t}=\frac{\partial}{\partial x_{j}}\left(T_{i j}{ }^{e l} \frac{\partial u_{i}}{\partial t}\right)-T_{i j}^{e l} \frac{\partial^{2} u_{i}}{\partial t \partial x_{j}}
$$

According to Royer and Dieulesaint [35], the Poynting vector is defined by:

$$
P_{j}=-T_{i j}{ }^{e l} \frac{\partial u_{i}}{\partial t}
$$

and verifies, for guided waves:

$$
\frac{\partial P_{j}}{\partial x_{j}}=c_{j} \frac{\partial e_{t o t}}{\partial x_{j}}
$$

where $c_{j}$ is the energy speed, i.e. the group velocity, in the direction $\mathbf{x}_{j}$ and $e_{t o t}=e_{c}+e_{p}$ is the bulk density of total energy within the structure. Moreover, because of the symmetry $S_{i j}=S_{j i}$ of the strain tensor, we can show that:

$$
T_{i j}^{e l} \frac{\partial^{2} u_{i}}{\partial t \partial x_{j}}=\frac{1}{2}\left(\lambda \delta_{i j}+2 \mu\right) \frac{\partial}{\partial t}\left(\frac{\partial u_{i}}{\partial x_{j}} \frac{\partial u_{j}}{\partial x_{i}}\right),
$$

which is the derivative of the bulk density of potential energy $e_{p}$.

Injecting equations $(\underline{B .6}),(\underline{B} .8)$ and $(\underline{B .9})$ in equation (B.5), we obtain:

$$
\frac{\partial e_{t o t}}{\partial t}+c_{j} \frac{\partial e_{t o t}}{\partial x_{j}}=\frac{\partial T_{i j}^{i n e l}}{\partial x_{j}} \frac{\partial u_{i}}{\partial t},
$$

where the last term can be developed using equation (B.4).

If we assume that the wave is longitudinal and propagates in direction $\mathbf{x}_{1}$ $\left(u_{2}=0\right)$, the wave displacement is:

$$
u_{1}=A_{1} \sin \left(\omega\left(t-x_{1} / c_{P}\right)\right)
$$


where $A_{1}$ is the amplitude, $\omega$ is the angular frequency and $c_{P}$ is the compressional wave speed. Thus we get:

$$
\frac{\partial T_{i j}{ }^{i n e l}}{\partial x_{j}} \frac{\partial u_{i}}{\partial t}=-(\chi+2 \eta) A_{1}^{2} \frac{\omega^{4}}{c_{P}^{2}} \cos ^{2}\left(\omega\left(t-x_{1} / c_{P}\right)\right) .
$$

If we remark that the bulk density of energy $e_{t o t}$ is equal to

$$
\rho\left(\frac{\partial u_{1}}{\partial t}\right)^{2}=\rho \omega^{2} A_{1}^{2} \cos ^{2}\left(\omega\left(t-x_{1} / c_{P}\right)\right)
$$

we obtain:

$$
\frac{\partial T_{i j}^{i n e l}}{\partial x_{j}} \frac{\partial u_{i}}{\partial t}=-(\chi+2 \eta) \frac{\omega^{2}}{\rho c_{P}^{2}} e_{t o t} .
$$

Using equations ( the equation of energy conservation of a longitudinal wave propagating in a viscoelastic solid is:

$$
\frac{\partial e_{t o t}}{\partial t}+\mathbf{v}_{\mathbf{g}} \cdot \nabla e_{t o t}=-\frac{e_{t o t}}{\tau}
$$

with $v_{g}=c_{P}$, the group speed and $\tau$, the characteristic time of energy dissipation [see e.g. 35, 43]:

$$
\tau=\frac{\rho c_{P}^{2}}{(\chi+2 \eta) \omega^{2}}=\frac{1}{\gamma v_{g}} .
$$

In equation (B.15), the term $-e_{\text {tot }} / \tau$ represents energy dissipation with time when the source force is not acting on the structure any more [e.g. 36]. Multiplying this equation by $\exp (t / \tau)$ gives:

$$
\left(\frac{\partial e_{t o t}}{\partial t}+\frac{e_{t o t}}{\tau}\right) \exp \left(\frac{t}{\tau}\right)+\mathbf{v}_{\mathbf{g}} \cdot \nabla e_{t o t} \exp \left(\frac{t}{\tau}\right)=0
$$

Writing $e_{t o t}^{\prime}=e_{t o t} \exp (t / \tau)$ leads to:

$$
\frac{\partial e_{t o t}^{\prime}}{\partial t}+\mathbf{v}_{\mathbf{g}} \cdot \nabla e_{t o t}^{\prime}=0
$$


Thus energy $e_{\text {tot }}^{\prime}=e_{\text {tot }} \exp (t / \tau)=e_{t o t} \exp (\gamma r)$ is conserved. Therefore, multiplying the energy by the factor $\exp (\gamma r)$ compensates the viscoelastic dissipation of energy with distance.

Note that if the wave is transversal and polarized along direction $\mathbf{x}_{2}$ and propagates along direction $\mathbf{x}_{1}$, we have:

$$
\frac{\partial T_{i j}{ }^{\text {inel }}}{\partial x_{j}} \frac{\partial u_{i}}{\partial t}=-\eta A_{2}^{2} \frac{\omega^{4}}{c_{S}^{2}} \cos ^{2}\left(\omega\left(t-x_{2} / c_{S}\right)\right),
$$

and we retrieve the conservation equation (B.15) with a different coefficient $\tau=\rho c_{S}^{2} / \eta \omega^{2}$, with $c_{S}$, the shear wave speed. Practically, the waves propagating in thin plates and thicks blocks are a complex combination of longitudinal and transversal waves. If we consider only one of these modes, either the mode $A_{0}$ of Lamb or the Rayleigh waves, the equation (B.15) of energy conservation is still verified provided that we integrate it over the depth [35] but the expression of the characteristic coefficient $\tau$ is much more complicated.

Here, we validate experimentally the model of energy attenuation in $\exp (-t / \tau)$ or in $\exp (-\gamma r)$ in the thin plates and the thick block investigated. To do so, we estimate the coefficient $\gamma$ by measuring the first arrival of the emitted vibration at different distances $r$ from an impact (Figure 18a) and filtering this vibration in different frequency ranges. For example in the PMMA plate, the squared amplitude of the $A_{0}$ mode decreases with distance $r$ as $\frac{1}{r} \exp (-\gamma r)$ (Figures 18b to 18d). We deduce the value of $\gamma$ as a function of frequency $f$ (Figure 18e).

When the first arrival can not be separated from the side reflections or when numerous side reflections occur in the structure after an impact, we can determine energy attenuation with an other method. For example on the glass plate, after an impact the envelope of the squared signal averaged 
811

812

over several periods decreases exponentially with time as:

$$
\overline{A(t)^{2}}=\overline{A(t=0)^{2}} \exp \left(-\frac{t}{\tau}\right)
$$

where $t=0$ is the impact time (Figure [19a). The characteristic time $\tau$ at frequency $f$ is simply the inverse of the slope of $\overline{A(t)^{2}}$ in semi-log scale, filtered in a frequency range centered on $f$ (Figures 19b to 19d). We thus show how the characteristic time $\tau$ decreases as the frequency $f$ increases (Figure 19p). Note that for a diffuse field, the inverse of $\tau$ is given by the average of the inverse of the characteristic times $\tau$ of each modes of propagation weighed by their percentage of partition. 


\section{Appendix C. Green's functions owing to a vertical load at the surface of an elastic half-space}

Here we recall the expression of the time Fourier's transform of the Green's function $\tilde{G}_{z z}(r, \omega)$ at the surface of a half-space owing to a vertical load on the surface.

Miller and Pursey [38] determined the exact expression of the surface vertical displacements $\tilde{U}_{z}(r, z, \omega)$ generated at a distance $r$ by a normal force $\mathbf{F}=\tilde{F}_{z}(\omega) \mathbf{u}_{z}$ on the surface of an elastic half-space [equation (72) of their paper with $z=0$ ]:

$$
\tilde{U}_{z}(r, \omega)=\frac{\tilde{F}_{z}(\omega) \xi^{2}}{\pi a \mu} \int_{0}^{+\infty} \frac{\sqrt{x^{2}-1}}{f_{0}(x)} J_{1}\left(k_{1} a x\right) J_{0}\left(k_{1} r x\right) \mathrm{d} x,
$$

where $a$ is the radius of the loading area, $\mu$ the Lamé shear modulus, $k_{1}=$ $\omega / c_{P}$, with the angular frequency $\omega=2 \pi f$ and the compressional wave speed $c_{P}, f_{0}(x)=\left(2 x^{2}-\xi^{2}\right)^{2}-4 x^{2} \sqrt{\left(x^{2}-1\right)\left(x^{2}-\xi^{2}\right)}, \xi=\sqrt{2(1-\nu) /(1-2 \nu)}$ and $\nu$ is Poisson's ratio. $J_{0}$ and $J_{1}$ are the Bessel's functions of the first kind.

For very small values of the radius of contact $a, J_{1}\left(k_{1} a x\right)$ can be approximated at a first order by $k_{1} a x / 2+O\left(a^{2}\right)$ so that

$$
\tilde{U}_{z}(r, \omega) \approx \frac{\tilde{F}_{z}(\omega) \xi^{2}}{2 \pi \mu} k_{1} \int_{0}^{+\infty} \frac{x \sqrt{x^{2}-1}}{f_{0}(x)} J_{0}\left(k_{1} r x\right) \mathrm{d} x .
$$

A first order approximation of the integral in equation (C.2) was calculated by Miller and Pursey [21] for large values of $k_{1} r=2 \pi f r / c_{P}$. From a practical viewpoint, this approximation is valid for impact problems because the impact generates high frequencies $1 \mathrm{kHz}<f<80 \mathrm{kHz}$ (Figures 6 $6 \mathrm{~d}, 8 \mathrm{k}$ and $7 \mathrm{~d})$ and $k_{1} r>>1$ even for small distances $r$ from the impact location. Using this computation, we can show that the vertical Green's 
${ }_{843}$ function $\tilde{G}_{z z}(r, \omega)=\tilde{U}_{z}(r, \omega) / \tilde{F}_{z}(\omega)$ is the sum of contributions of compres${ }_{844}$ sional, shear and Rayleigh waves, respectively, $\tilde{G}_{z z}^{P}, \tilde{G}_{z z}^{S}$ and $\tilde{G}_{z z}^{R}$ :

845

846

847

848

849

$$
\tilde{G}_{z z}=\tilde{G}_{z z}^{P}+\tilde{G}_{z z}^{S}+\tilde{G}_{z z}^{R}
$$

with

$$
\begin{aligned}
\tilde{G}_{z z}^{P}(r, \omega) & \approx-\frac{i}{\mu} A_{P} \frac{k_{1}}{\left(k_{1} r\right)^{2}} \exp \left(-i \omega r / c_{P}\right) \\
\tilde{G}_{z z}^{S}(r, \omega) & \approx-\frac{i}{\mu} A_{S} \frac{k_{1}}{\left(k_{1} r\right)^{2}} \exp \left(-i \omega r / c_{S}\right) \\
\tilde{G}_{z z}^{R}(r, \omega) & \approx-\frac{i}{\mu} A_{R} k_{1} \sqrt{\frac{2}{\pi k_{1} r}} \exp \left(-i\left(\omega r / c_{R}-\frac{\pi}{4}\right)\right),
\end{aligned}
$$

where $c_{P}, c_{S}$ and $c_{R}$ are the compressional, shear and Rayleigh waves speeds, respectively, and where $A_{P}, A_{S}$ and $A_{R}$ are only functions of Poisson's ratio $\nu$ (Figure 201):

$$
\begin{aligned}
A_{P}(\nu) & =\frac{\xi^{2}}{2 \pi\left(2-\xi^{2}\right)^{2}}, \\
A_{S}(\nu) & =\frac{2\left(\xi^{2}-1\right)}{\pi \xi^{3}}, \\
A_{854}(\nu) & =\frac{\xi^{2}}{2} \frac{\sqrt{x_{0}\left(x_{0}^{2}-1\right)}}{f_{0}^{\prime}\left(x_{0}\right)},
\end{aligned}
$$

with $x_{0}$, the real positive root of $f_{0}(x)$. 


\section{Appendix D. Detailed calculation of coefficient $\beta$}

We detail here the calculation of the coefficient $\beta$ that appears in the expression of the elastic energy $W_{e l}$ radiated in a block [equation (35)]. $\beta$ is defined as the imaginary part of

$$
\int_{0}^{X} \frac{x \sqrt{x^{2}-1}}{f_{0}(x)} \mathrm{d} x
$$

where $f_{0}(x)=\left(2 x^{2}-\xi^{2}\right)^{2}-4 x^{2} \sqrt{\left(x^{2}-1\right)\left(x^{2}-\xi^{2}\right)}, \xi=\sqrt{2(1-\nu) /(1-2 \nu)}$ and $\nu$ the Poisson ratio of the block. $X$ is a number greater than the real root $x_{0}$ of $f_{0}$, which is represented in Figure 5 .

Let the function $f$ be:

$$
f: x \longrightarrow \frac{x \sqrt{x^{2}-1}}{\left(2 x^{2}-\xi^{2}\right)^{2}-4 x^{2} \sqrt{\left(x^{2}-1\right)\left(x^{2}-\xi^{2}\right)}} .
$$

For most materials, the Poisson ratio $\nu$ is between 0 and 0.5 , corresponding to values of $\xi$ from 1.4 to 10 . To calculate $\beta$ we have to look at the definition of $f$ over the intervals $[0,1[,[1, \xi[$ and $x \geq \xi$ :

- For $x \in\left[0,1\left[, x^{2}-1<0\right.\right.$ and $x^{2}-\xi^{2}<0$, then we can then write $\sqrt{x^{2}-1}=i \sqrt{1-x^{2}}$ and $\sqrt{x^{2}-\xi^{2}}=i \sqrt{\xi^{2}-x^{2}}$ where $i$ is the complex number $\sqrt{-1}$. Over this interval, $f(x)$ is a pure imaginary number:

$$
f(x)=\frac{i x \sqrt{1-x^{2}}}{\left(2 x^{2}-\xi^{2}\right)^{2}+4 x^{2} \sqrt{\left(1-x^{2}\right)\left(\xi^{2}-x^{2}\right)}}
$$

and

$$
\operatorname{Im}(f(x))=f_{1}(x)=\frac{x \sqrt{1-x^{2}}}{\left(2 x^{2}-\xi^{2}\right)^{2}+4 x^{2} \sqrt{\left(1-x^{2}\right)\left(\xi^{2}-x^{2}\right)}} .
$$

Regardless of the value of $\xi, f_{1}$ is continuous over $[0,1]$ with $f_{1}(0)=$ $f_{1}(1)=0$ and $f_{1}$ is $C^{\infty}$ over $[0,1[$. 
- For $x \in\left[1, \xi\left[, x^{2}-1>0\right.\right.$ and $x^{2}-\xi^{2}<0$, therefore $\sqrt{x^{2}-\xi^{2}}=$ $i \sqrt{\xi^{2}-x^{2}}$. Over this interval:

$$
f(x)=\frac{x \sqrt{x^{2}-1}}{\left(2 x^{2}-\xi^{2}\right)^{2}-4 i x^{2} \sqrt{\left(x^{2}-1\right)\left(\xi^{2}-x^{2}\right)}} .
$$

Multiplying the numerator and the denominator by the complex conjugate of the denominator leads to:

$$
f(x)=\frac{x \sqrt{x^{2}-1}\left[\left(2 x^{2}-\xi^{2}\right)^{2}+4 i x^{2} \sqrt{\left(x^{2}-1\right)\left(\xi^{2}-x^{2}\right)}\right]}{\left(2 x^{2}-\xi^{2}\right)^{4}+16 x^{4}\left(x^{2}-1\right)\left(\xi^{2}-x^{2}\right)}
$$

and

$$
\operatorname{Im}(f(x))=f_{2}(x)=\frac{4 x^{3}\left(x^{2}-1\right) \sqrt{\xi^{2}-x^{2}}}{\left(2 x^{2}-\xi^{2}\right)^{4}+16 x^{4}\left(x^{2}-1\right)\left(\xi^{2}-x^{2}\right)} .
$$

Regardless of the value of $\xi, f_{2}$ is continuous over $[1, \xi]$ with $f_{2}(1)=$ $f_{2}(\xi)=0$ and $f_{2}$ is $C^{\infty}$ function over $[1, \xi[$.

- For $x \geq \xi, x^{2}-1>0$ and $x^{2}-\xi^{2}>0$, therefore $f$ is a real function over this interval and its imaginary part is null, except for the contribution of the pole $x_{0}$ of $f_{0}$, which is always greater than $\xi$ (Figure 5). The integral of $f$ over this interval is due to half of its residue in $x_{0}$ :

$$
\int_{\xi}^{X} f(x) d x=-i \pi \frac{x_{0} \sqrt{x_{0}^{2}-1}}{f_{0}^{\prime}\left(x_{0}\right)}
$$

Finally, $\beta=\int_{0}^{1} f_{1}(x) \mathrm{d} x+\int_{1}^{\xi} f_{2}(x) \mathrm{d} x-\pi \frac{x_{0} \sqrt{x_{0}^{2}-1}}{f_{0}^{\prime}\left(x_{0}\right)} . \quad \beta$ is represented as a function of the Poisson ratio $\nu$ in Figure 21. 


\section{References}

[1] H. Lamb, On the propagation of tremors over the surface of an elastic solid, Philos. T Roy. Soc. A 203 (1904) 1-42. doi:10.1098/rsta.1904.0013.

[2] H. Kanamori, The energy release in great earthquakes, J. Geophys. Res. 82 (1977) 2981-2987. doi:10.1029/JB082i020p02981.

[3] K. Aki, P. Richards, Quantitative Seismology : Theory and Methods, volume 1, W.H. Freeman, 1980.

[4] X. Pérez-Campos, G. C. Beroza, An apparent mechanism dependence of radiated seismic energy, J. Geophys. Res. 106 (2001) 11127-11136. doi:10.1029/2000JB900455.

[5] M. Lancieri, R. Madariaga, F. Bonilla, Spectral scaling of the aftershocks of the Tocopilla 2007 earthquake in northern Chile, Geophys. J. Int. 189 (2012) 469-480. doi:10.1111/j.1365-246X.2011.05327.x.

[6] J. B. Fletcher, A. McGarr, Moments, magnitudes, and radiated energies of non-volcanic tremor near Cholame, CA, from ground motion spectra at UPSAR, Geophys. Res. Lett. 38 (2011) L16314. doi:10.1029/2011GL048636.

[7] J. Deparis, D. Jongmans, F. Cotton, L. Baillet, F. Thouvenot, D. Hantz, Analysis of rock-fall and rock-fall avalanche seismograms in the French Alps, Bull. Seism. Soc. Am. 98 (2008) 1781-1796. doi:10.1785/0120070082. 
[8] I. Vilajosana, E. Suriñach, A. Abellan, G. Khazaradze, D. Garcia, J. Llosa, Rockfall induced seismic signals: case study in Montserrat, Catalonia, Nat. Hazards Earth Syst. Sci 8 (2008) 805-812. doi:10.5194/nhess-8-805-2008.

[9] C. Hibert, A. Mangeney, G. Grandjean, N. M. Shapiro, Slope instabilities in Dolomieu crater, Réunion Island: From seismic signals to rockfall characteristics, J. Geophys. Res. 116 (2011) F04032. doi:10.1029/2011JF002038.

[10] C. Hibert, A. Mangeney, G. Grandjean, C. Baillard, D. Rivet, N. M. Shapiro, C. Satriano, A. Maggi, P. Boissier, V. Ferrazzini, W. Crawford, Automated identification, location, and volume estimation of rockfalls at piton de la fournaise volcano, J. Geophys. Res. 119 (2014) 1082-1105. doi:10.1002/2013JF002970.

[11] L. Moretti, A. Mangeney, Y. Capdeville, E. Stutzmann, C. Huggel, D. Schneider, F. Bouchut, Numerical modeling of the Mount Steller landslide flow history and of the generated long period seismic waves, Geophys. Res. Lett. 39 (2012). doi:10.1029/2012GL052511.

[12] V. C. Tsai, B. Minchew, M. P. Lamb, J.-P. Ampuero, A physical model for seismic noise generation from sediment transport in rivers, Geophys. Res. Lett. 39 (2012). doi 10.1029/2011GL050255.

[13] O. Lengliné, J. E. Elkhoury, G. Daniel, J. Schmittbuhl, R. Toussaint, J. P. Ampuero, M. Bouchon, Interplay of seismic and aseismic deformations during earthquake swarms: An experimen- 
tal approach, Earth Planet. Sc. Lett. 331-332 (2012) 215-223. doi:10.1016/j.epsl.2012.03.022.

[14] M. Stojanova, S. Santucci, L. Vanel, O. Ramos, High frequency monitoring reveals aftershocks in subcritical crack growth, Phys. Rev. Lett. 112 (2014) 115502. doi:10.1103/PhysRevLett.112.115502.

[15] G. Michlmayr, D. Cohen, D. Or, Sources and characteristics of acoustic emissions from mechanically stressed geologic granular media - A review, Earth Sc. Rev. 112 (2012) 97-114. doi:10.1016/j.earscirev.2012.02.009.

[16] P. A. Houle, J. P. Sethna, Acoustic Emission from crumpling paper, Phys. Rev. E 54 (1995). doi:S1063-651X9604307-3.

[17] J. P. Sethna, K. A. Dahmen, C. R. Myers, Crackling Noise, Nature 410 (2001) 242-250. doi $10.1038 / 35065675$.

[18] D. J. Buttle, C. B. Scruby, Characterization of particle impact by quantitative acoustic emission, Wear 137 (1990) 63-90. doi:10.1016/0043-1648(90)90018-6.

[19] D. J. Buttle, S. R. Martin, C. B. Scruby, Particle sizing by quantitative acoustic emission, Res. Nondestruct. Eval. 3 (1991) 1-26. doi:10.1007/BF01606508.

[20] S. Crampin, Higher modes of seismic surface waves: Second Rayleigh mode energy, J. Geophys. Res. 70 (1965) 5135-5143. doi:10.1029/JZ070i020p05135. 
[21] G. F. Miller, H. Pursey, On the partition of energy between elastic waves in a semi-infinite solid, Proc. R. Soc. Lond. A. Mat. 233 (1955) 55-69. doi:10.1098/rspa.1955.0245.

[22] H. Goyder, R. G. White, Vibrational power flow from machines into built-up structures, part I: introduction and approximate analyses of beam and plate-like foundations, J. Sound Vib. 68 (1980) 59-75. doi: $10.1016 / 0022-460 X(80) 90452-6$.

[23] R. L. Weaver, On diffuse waves in solid media, J. Acoust. Soc. Am. 71 (1982). doi:10.1121/1.387816.

[24] R. L. Weaver, Diffuse waves in finite plates, J. Sound Vib. 94 (1984) 319-335. doi:10.5194/nhess-8-805-2008.

[25] R. L. Weaver, Diffuse elastic waves at a free surface, J. Acoust. Soc. Am. 78 (1985). doi:10.1121/1.392576.

[26] K. Mayeda, L. Malagnini, Source radiation invariant property of local and near-regional shear-wave coda: Application to source scaling for the mw 5.9 wells, nevada sequence, Geophys. Res. Lett. 37 (2010). doi:10.1029/2009GL042148.

[27] R. M. Davies, The determination of static and dynamic yield stresses using a steel ball, P. Roy. Soc. Lond. A Mat. 197 (1949) 416-432. doi:10.1098/rspa.1949.0073.

[28] E. Falcon, C. Laroche, S. Fauve, C. Coste, Behavior of one inelastic ball bouncing repeatedly off the ground, Eur. Phys. J. B 3 (1998) 45-57. doi:10.1007/s100510050283. 
[29] H. Hertz, Über die Berührung fester elastischer Körper (On the vibration of solid elastic bodies), J. Reine Angew. Math. 92 (1882) 156-171. doi:10.1515/crll.1882.92.156.

[30] K. Johnson, Contact Mechanics, Cambridge University Press, 1985.

[31] G. C. McLaskey, S. D. Glaser, Hertzian impact: Experimental study of the force pulse and resulting stress waves, J. Acoust. Soc. Am. 128 (2010) 1087. doi:10.1121/1.3466847.

[32] S. C. Hunter, Energy absorbed by elastic waves during impact, J. Mech. Phys. Solids 5 (1957) 162-171. doi:10.1016/0022-5096(57)90002-9.

[33] J. Reed, Energy losses due to elastic wave propagation during an elastic impact, J. Phys. D Appl. Phys. 18 (1985) 2329. doi:10.1088/0022-3727/18/12/004.

[34] I. M. Hutchings, Energy absorbed by elastic waves during plastic impact, J. Phys. D: Appl. Phys. 12 (1979) 1819. doi:10.1016/0022-5096(57)90002-9.

[35] D. Royer, E. Dieulesaint, Elastic Waves in Solids I: Free and Guided Propagation, Springer, 2000.

[36] O. M. Bouthier, R. J. Bernhard, Simple models of the energetics of transversely vibrating plates, J. Sound Vib. 182 (1995) 149-164. doi:10.1006/jsvi.1995.0187.

[37] J. Michaels, T. E. Michaels, W. Sachse, Applications of deconvolution to acoustic emission signal analysis, Mater. Eval. 39 (1981) 1032-1036. 
[38] G. F. Miller, H. Pursey, The field and radiation impedance of mechanical radiators on the free surface of a semi-infinite isotropic solid, Proc. R. Soc. Lond. A. Mat. 223 (1954) 521-541. doi:10.1098/rspa.1954.0134.

[39] E. Kausel, Lamb's problem at its simplest, Proc. R. Soc. Lond. A. Mat. 469 (2013). doi:10.1098/rspa.2012.0462.

[40] F. J. Sánchez-Sesma, R. L. Weaver, H. Kawase, S. Matsushima, F. Luzon, M. Campillo, Energy Partitions among Elastic Waves for Dynamic Surface Loads in a Semi-Infinite Solid, Bull. Seism. Soc. Am. 101 (2011) 1704-1709. doi:10.1785/0120100196.

[41] M. Farin, Études expérimentales de la dynamique et de l'émission sismique des instabilités gravitaires, Ph.D. thesis, IPGP, Paris, 2015.

[42] R. Ramírez, T. Pöschel, N. V. Brilliantov, T. Schwager, Coefficient of restitution of colliding viscoelastic spheres, Phys. Rev. E 60 (1999) 4465-4472. doi:10.1103/PhysRevE.60.4465.

[43] L. Landau, E. Lifshitz, Theory of Elasticity, volume 7, Pergamon Press, 1970.

[44] A. Fuegel, Statistical calculation and development of glass properties, http://glassproperties.com/, 2007. (Accessed 10 December 2014).

[45] MIT, Material properties database, http://www.mit.edu/ 6.777/ matprops/pmma.htm, 2014. (Accessed 10 December 2014).

[46] G. Elert, The physics factbook, http://hypertextbook.com/facts/ 1999/KatrinaJones.shtml, 1999. (Accessed 10 December 2014). 
Table 2: Physical values used for calculation of the radiated elastic energy in the glass plate and the concrete block: density $\rho$, Young's modulus $E$, Poisson ratio $\nu$, compressional and shear wave speeds $c_{P}$ and $c_{S}$, bending stiffness $B$, characteristic distance $1 / \gamma$ and time $\tau$ of energy attenuation, group velocity $v_{g}$ (that depends on the frequency $f\left(\right.$ in Hz)), phase velocity $v_{\phi}$ and coefficient $\beta$. Glass parameters are from Fuegel [4] and PMMA parameters from the MIT material properties database 45]. Elastic parameters $E$ and $\nu$ of concrete are estimated from the compressional and shear wave velocities measured through the block and the density $\rho$ of concrete is from Elert [46].

\begin{tabular}{|c|c|c|c|c|c|c|c|c|c|c|c|c|}
\hline material & & $\begin{array}{c}\rho \\
\left(\mathrm{kg} \mathrm{m}^{-3}\right) \\
\end{array}$ & $\begin{array}{c}E \\
(\mathrm{GPa})\end{array}$ & $\begin{array}{l}\nu \\
- \\
\end{array}$ & $\begin{array}{c}c_{P} \\
\left(\mathrm{~m} \mathrm{~s}^{-1}\right)\end{array}$ & $\begin{array}{c}c_{S} \\
\left(\mathrm{~m} \mathrm{~s}^{-1}\right)\end{array}$ & $\begin{array}{c}B \\
(\mathrm{~J}) \\
\end{array}$ & $\begin{array}{c}\gamma \\
(1 / \mathrm{m}) \\
\end{array}$ & $\begin{array}{c}\tau \\
(\mathrm{s}) \\
\end{array}$ & $\begin{array}{c}v_{g} \\
\left(\mathrm{~m} \mathrm{~s}^{-1}\right)\end{array}$ & $\begin{array}{c}v_{\phi} \\
\left(\mathrm{m} \mathrm{s}^{-1}\right)\end{array}$ & $\begin{array}{l}\beta \\
- \\
\end{array}$ \\
\hline glass & $\begin{array}{l}k h<1 \\
k h>1\end{array}$ & 2500 & 74 & 0.2 & 5730 & 3500 & 4760 & $\begin{array}{c}0.014 f^{1 / 6} \\
8.5 \times 10^{-5} f^{2 / 3}\end{array}$ & $3.8 f^{-2 / 3}$ & $\begin{array}{c}18.6 f^{1 / 2} \\
3100\end{array}$ & $\begin{array}{c}9.3 f^{1 / 2} \\
3100\end{array}$ & - \\
\hline PMMA & $\begin{array}{l}k h<1 \\
k h>1\end{array}$ & 1180 & 4.4 & 0.37 & 1920 & 860 & 357 & $\begin{array}{c}1 \\
4.8 \times 10^{-3} f^{2 / 3}\end{array}$ & $\begin{array}{l}0.09 f^{-1 / 2} \\
0.15 f^{-2 / 3}\end{array}$ & $\begin{array}{c}11.7 f^{1 / 2} \\
1400\end{array}$ & $\begin{array}{c}5.8 f^{1 / 2} \\
1400\end{array}$ & - \\
\hline concrete & - & 2200 & 16.3 & 0.4 & 4030 & 1620 & - & $2.3 \times 10^{-5} f$ & $28 f^{-1}$ & 1530 & 1530 & 0.3 \\
\hline
\end{tabular}




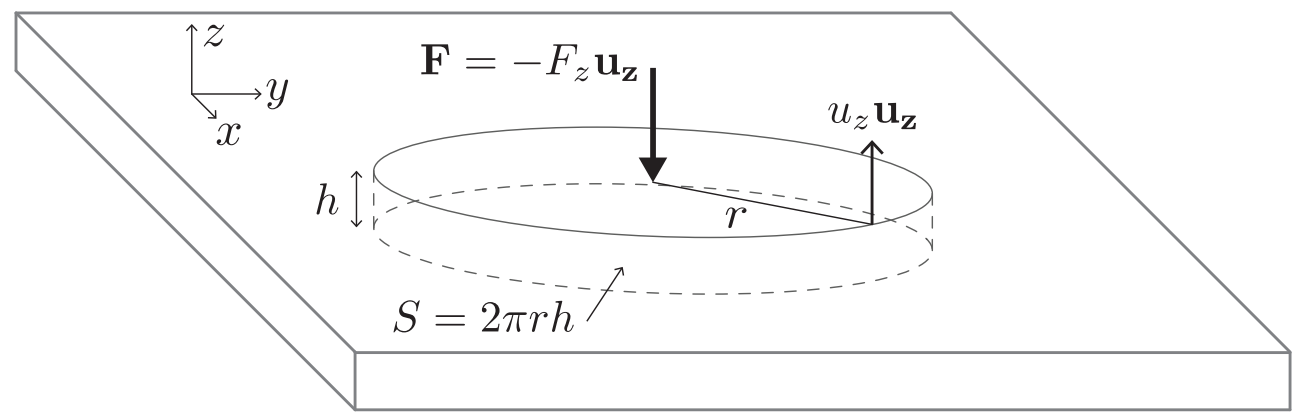

Figure 1: Sketch of the thin plate of thickness $h$, characterized by Cartesian coordinates $x, y, z . z=0$ corresponds to the plate free surface. When a normal impact force $-F_{z} \mathbf{u}_{z}$ excites the plate at the origin $(0,0,0)$, Lamb waves are emitted radially and generate a displacement field $\mathbf{u} \approx u_{z}(r, t) \mathbf{u}_{z} . S$ is a closed section of the plate, surrounding the impact position and corresponds here to a cylinder of radius $r$ and height equal to the plate thickness $h$. 

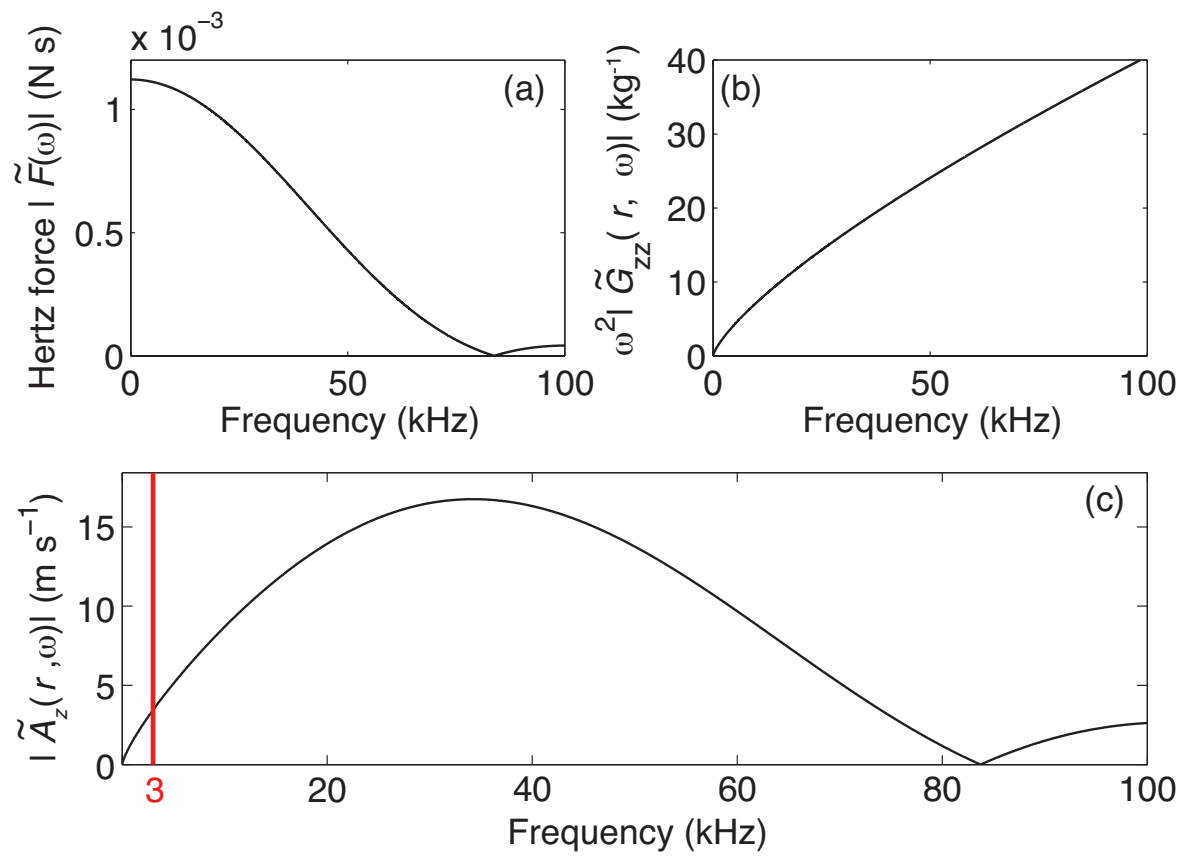

Figure 2: (a) Fourier transform $|\tilde{F}(\omega)|$ of the ideal Hertz's force of elastic impact of a 4-mm diameter steel sphere on PMMA. (b) Green's function $\left|\tilde{G}_{z z}(r, \omega)\right|$ [equation (12)] at $r=10 \mathrm{~cm}$ multiplied by $\omega^{2}$ (c) Synthetic amplitude spectrum $\left|\tilde{A}_{z}(r, \omega)\right|$ obtained by the product of the force in (a) and the function in (b). 


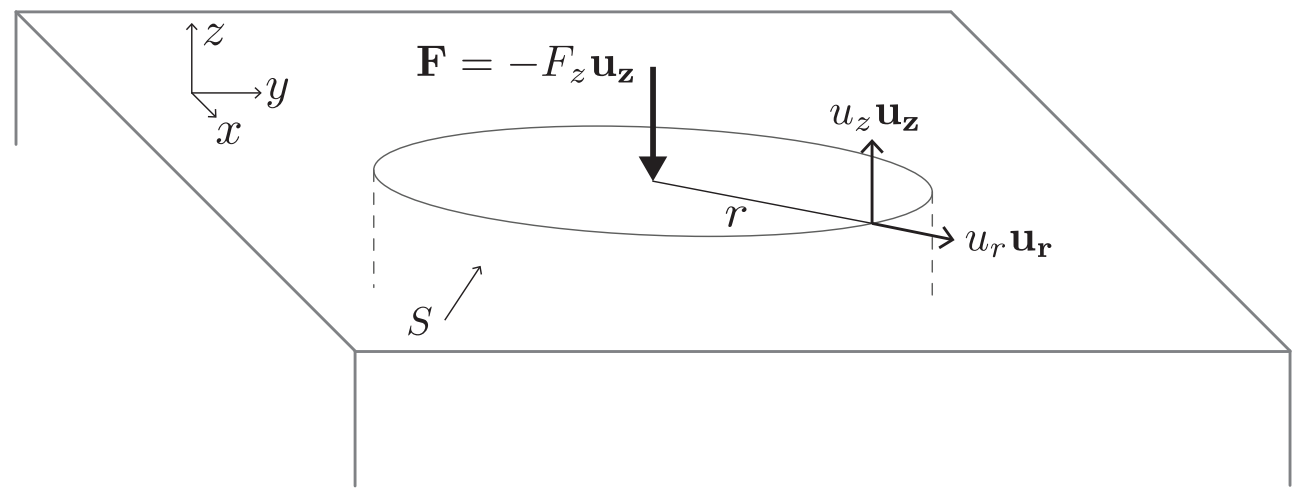

Figure 3: Sketch of the thick block configuration, characterized by Cartesian coordinates $x, y, z . z=0$ corresponds to the block free surface. When a normal impact force $-F_{z} \mathbf{u}_{z}$ excites the block normally at the origin $(0,0,0)$, Rayleigh waves are emitted radially at the surface and generate a displacement field $\mathbf{u}=u_{r}(r, z, t) \mathbf{u}_{r}+u_{z}(r, z, t) \mathbf{u}_{z}$ with an amplitude that decreases exponentially with depth $z$ (see text). $S$ is a closed section of the block, surrounding the impact position, and corresponds here to a cylinder of radius $r$ and infinite height. 

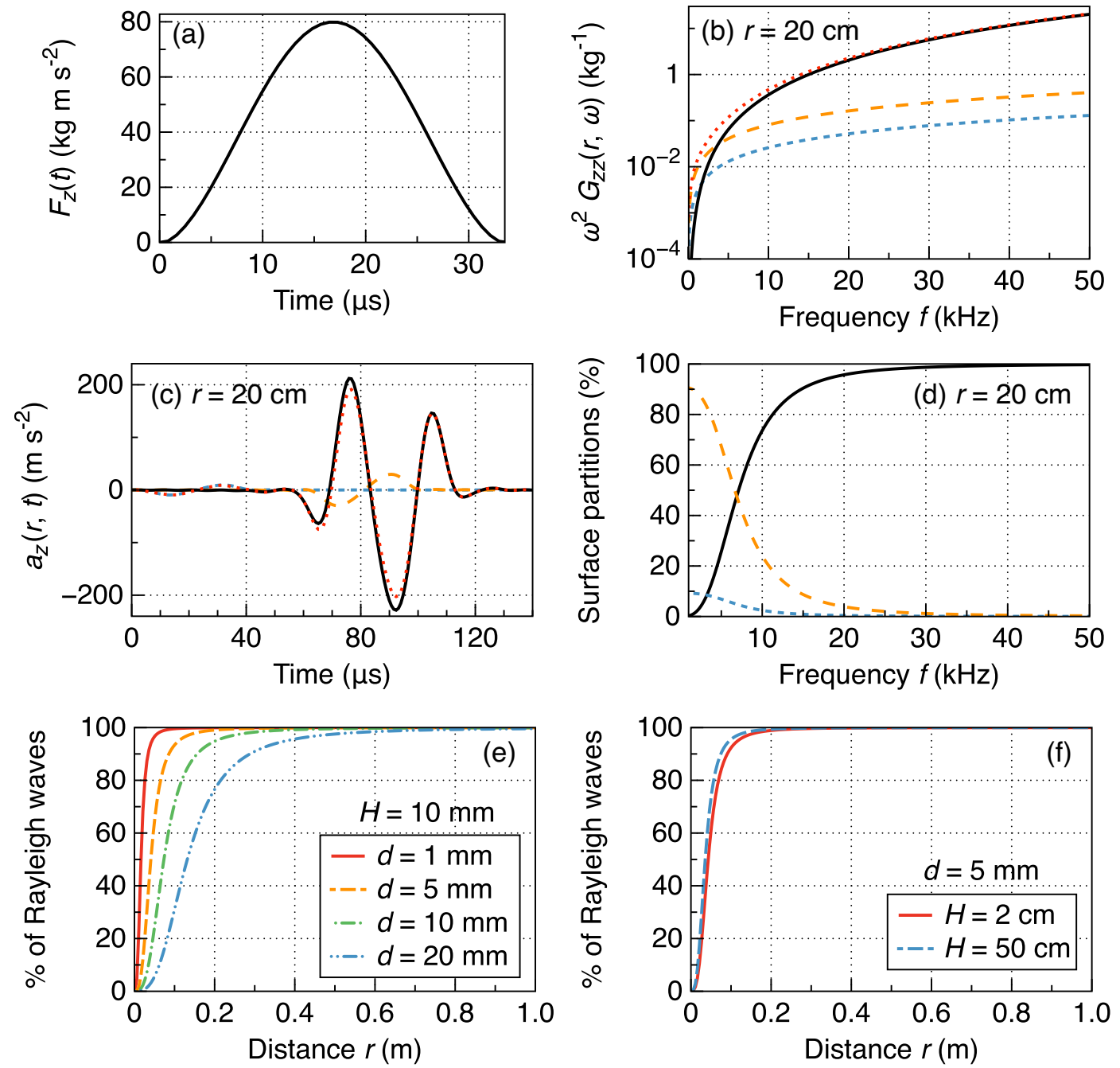

Figure 4: (a) Hertz's force of elastic impact of a steel bead of diameter $d=5 \mathrm{~mm}$ dropped from height $H=10 \mathrm{~cm}$ on a concrete block is convolved with (b) the Green's functions $\tilde{G}_{z z}^{P}, \tilde{G}_{z z}^{S}$ and $\tilde{G}_{z z}^{R}$ [equations (19), (20) and (21), respectively], multiplied by $\omega^{2}$, at $r=20$ $\mathrm{cm}$ from the impact to obtain (c) the synthetic vertical vibration acceleration $a_{z}(r, t)$ of each mode at the surface. (d) Percentage of the energy transported by compressional, shear and Rayleigh waves at $r=20 \mathrm{~cm}$ from the impact as a function of frequency $f$. (e) Percentage $\pi_{\text {surf }}^{R}(r)$ of Rayleigh waves in the surface vibration as a function of the distance $r$ from the impact for (e) a fall height $H=10 \mathrm{~cm}$ and different bead diameters $d$ and (f) for a bead diameter of $d=5 \mathrm{~mm}$ and fall heights $H=5 \mathrm{~cm}$ and $H=50 \mathrm{~cm}$. 


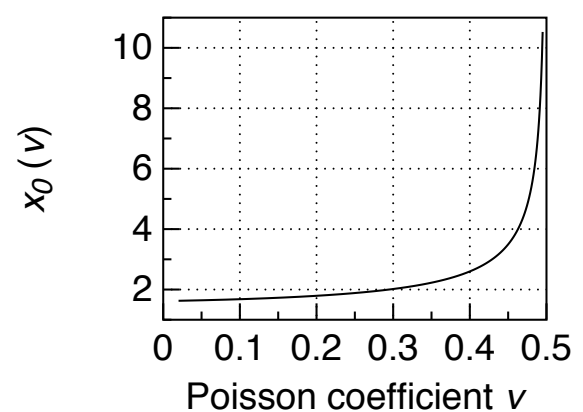

Figure 5: Value of the real solution $x_{0}$ of $f_{0}(x)=0$ as a function of Poisson's ratio $\nu$. 

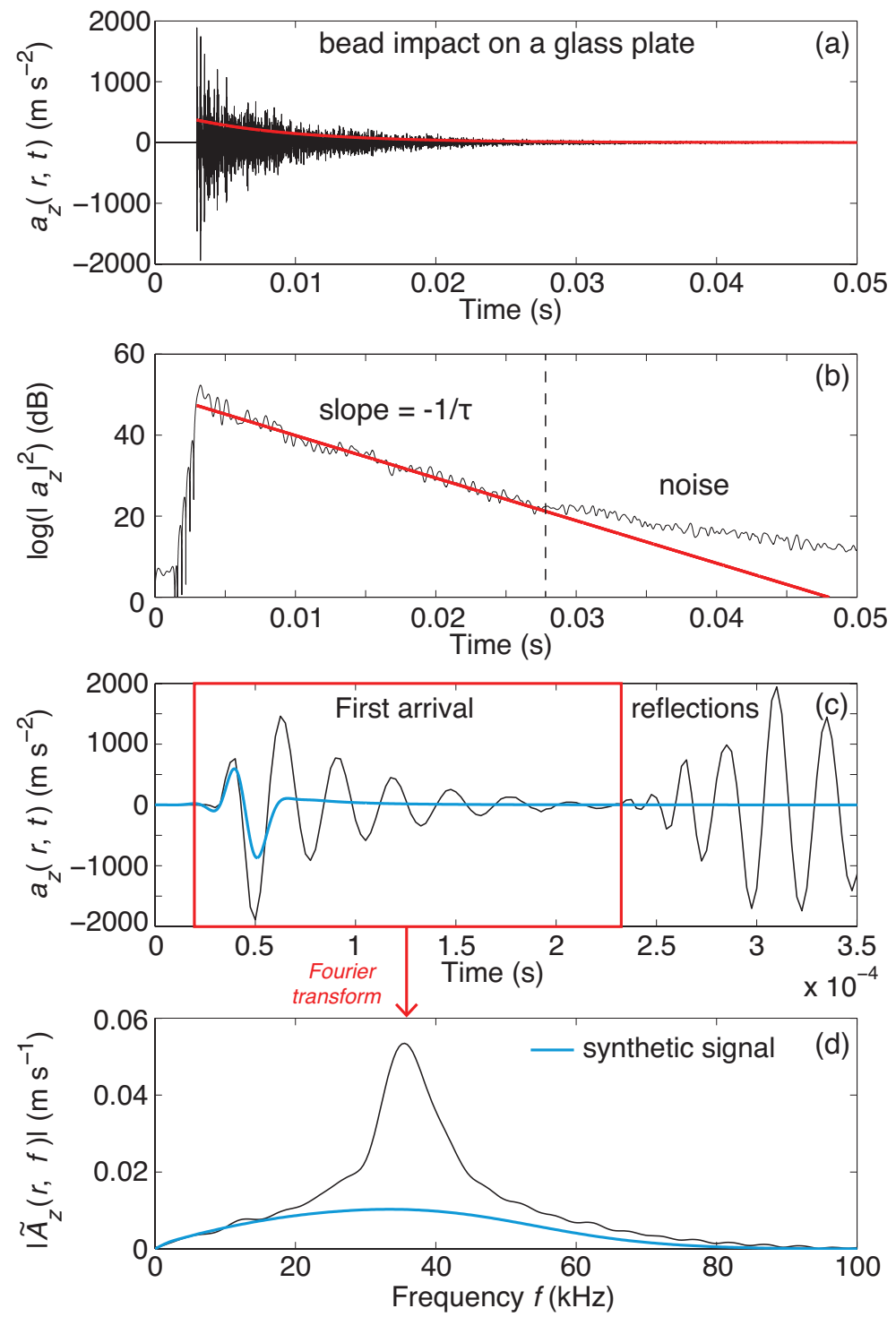

Figure 6: (a)-(c) Normal surface acceleration $a_{z}(r, t)$, filtered below $100 \mathrm{kHz}$, recorded at $r=6 \mathrm{~cm}$ from the source after the impact of a steel bead of diameter $4 \mathrm{~mm}$ on the glass plate. (a) and (b) The wave reflects many times off the plate lateral sides and the energy decreases exponentially with time due to viscoelastic dissipation (red line). In (b), $a_{z}(r, t)$ is squared, filtered below $2000 \mathrm{~Hz}$ and plotted in semi-log form. (c) The plate is sufficiently large to record the first wave arrival entirely (red frame) before the return of the first side reflections. (d) Time Fourier transform $\left|\tilde{A}_{z}(r, f)\right|$ of the first wave arrival as a function of frequency $f$. The thick blue lib 9 in (c) and (d) is a synthetic signal obtained with the convolution of the Green's function in equation (12) with the force of Hertz. The discrepancy of the measured signal with theory is discussed in section 4 . 

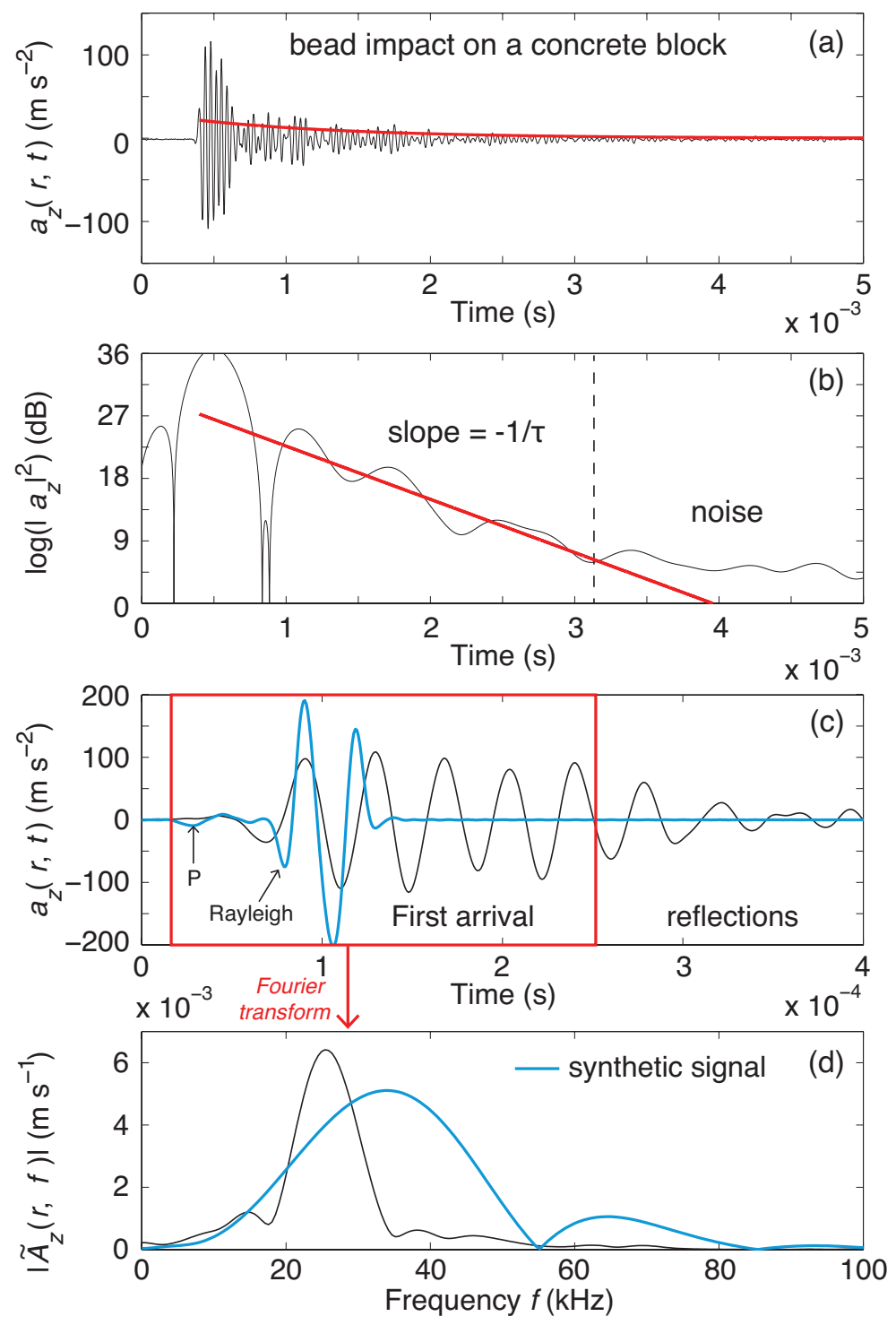

Figure 7: (a)-(c) Normal surface acceleration $a_{z}(r, t)$ recorded at $r=20 \mathrm{~cm}$ from the source after the impact of a steel bead of diameter $5 \mathrm{~mm}$ on the concrete block. (a) and (b) The wave reflects several times off the block boundaries and the energy decreases exponentially with time due to viscoelastic dissipation (red line). In (b), $a_{z}(r, t)$ is squared, filtered below $2000 \mathrm{~Hz}$ and plotted in semi-log form. (c) The block is sufficiently large to record most of the first wave arrival (red frame) before the return of the first side reflection that should arrive on the right side of the red frame. (d) Time Fourier transform $\left|\tilde{A}_{z}(r, f)\right|$ of the first wave arrival as a function of frequency $f$. The thick blue line in (c) and (d) is a synthetic signal obtained with the convolution of the Green's function in equation (18) with the force of Hertz. In the temporal synthetic signal in (c), we can discern the compressional wave (noted $P$ ) and the Rayleigh waves. The discrepancy of the measured signal with theory is discussed in section 4 . 

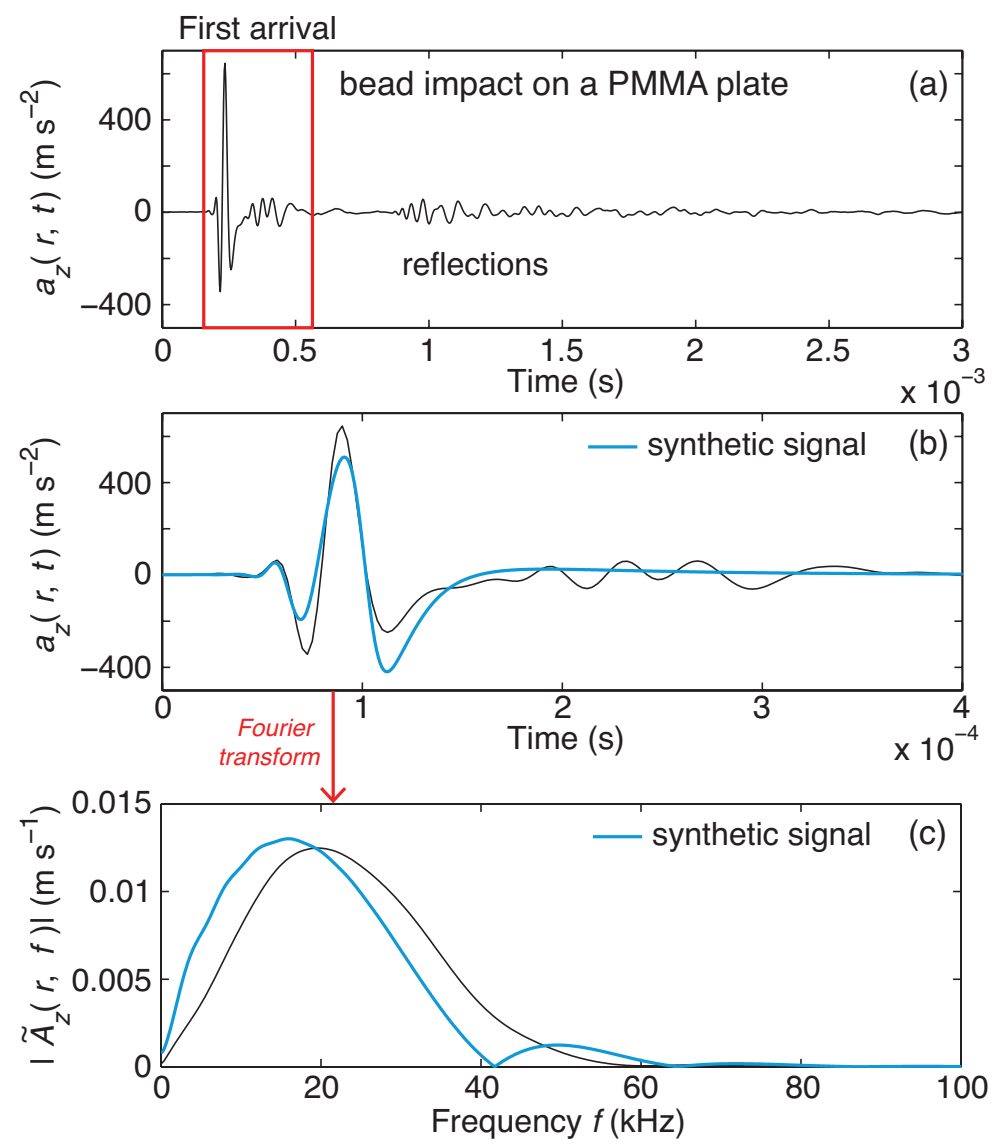

Figure 8: (a) and (b) Normal surface acceleration $a_{z}(r, t)$, filtered below $100 \mathrm{kHz}$, recorded at $r=10 \mathrm{~cm}$ from the source after the impact of a steel bead of diameter $3 \mathrm{~mm}$ on the PMMA plate. (a) The direct wave front (red frame) is clearly separated from its reflections off the plate lateral sides. (b) Zoom on the first wave arrival. (c) Time Fourier transform $\left|\tilde{A}_{z}(r, f)\right|$ of the first wave arrival as a function of the frequency $f$. The thick blue line in (b) and (c) is a synthetic signal obtained with the convolution of the Green's function in equation (12) with the force of Hertz. The discrepancy of the measured signal with theory is discussed in section 4 


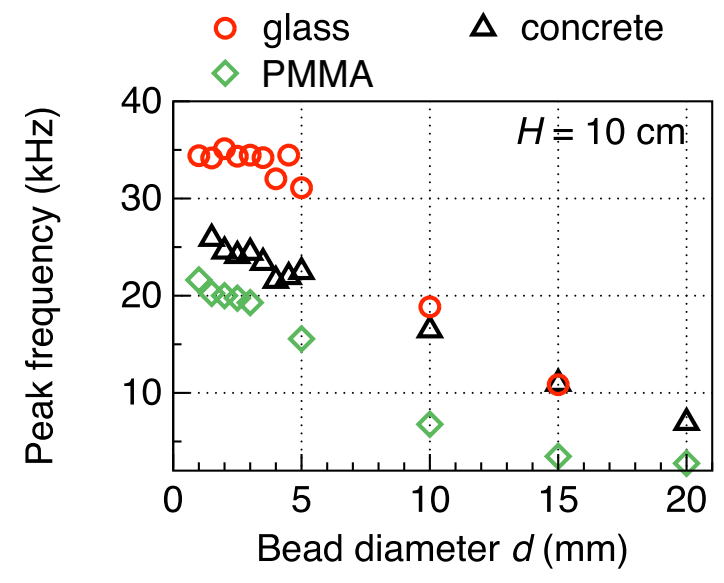

Figure 9: Frequency of the maximum of the amplitude spectrum $\left|\tilde{A}_{z}(r, f)\right|$, or peak frequency, for impacts of steel beads of different bead diameters $d$ on the glass plate, PMMA plate and concrete block. The peak frequency is independent of the fall height in the range investigated. 

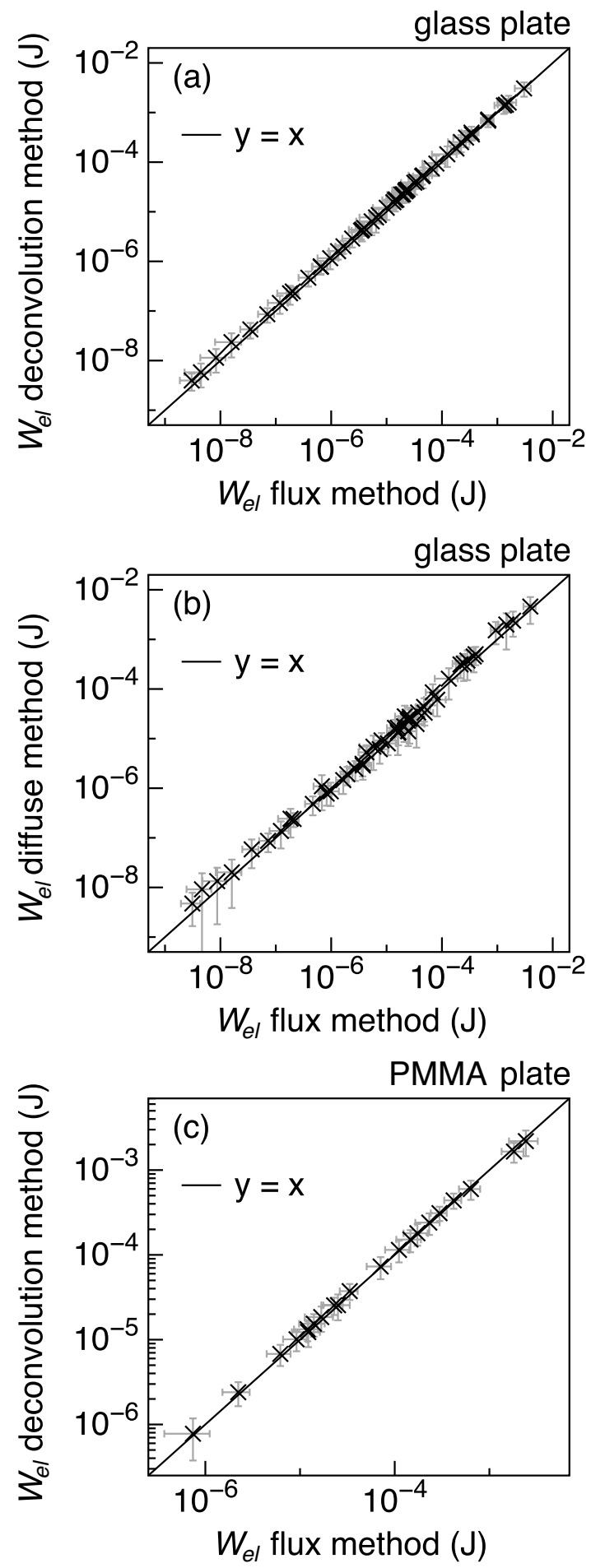

Figure 10: Comparison of the radiated elast63energy $W_{e l}$ calculated using the three methods [equations (8), (13) and (17)] for impacts of steel beads of various diameters from 1 $\mathrm{mm}$ to $20 \mathrm{~mm}$ dropped from various heights from $2 \mathrm{~cm}$ to $25 \mathrm{~cm}$ on (a) and (b) the glass plate and (c) the PMMA plate. Error bars ( \pm 1 standard deviation) are estimated from reproducibility tests conducted on a series of 12 identical experiments. 

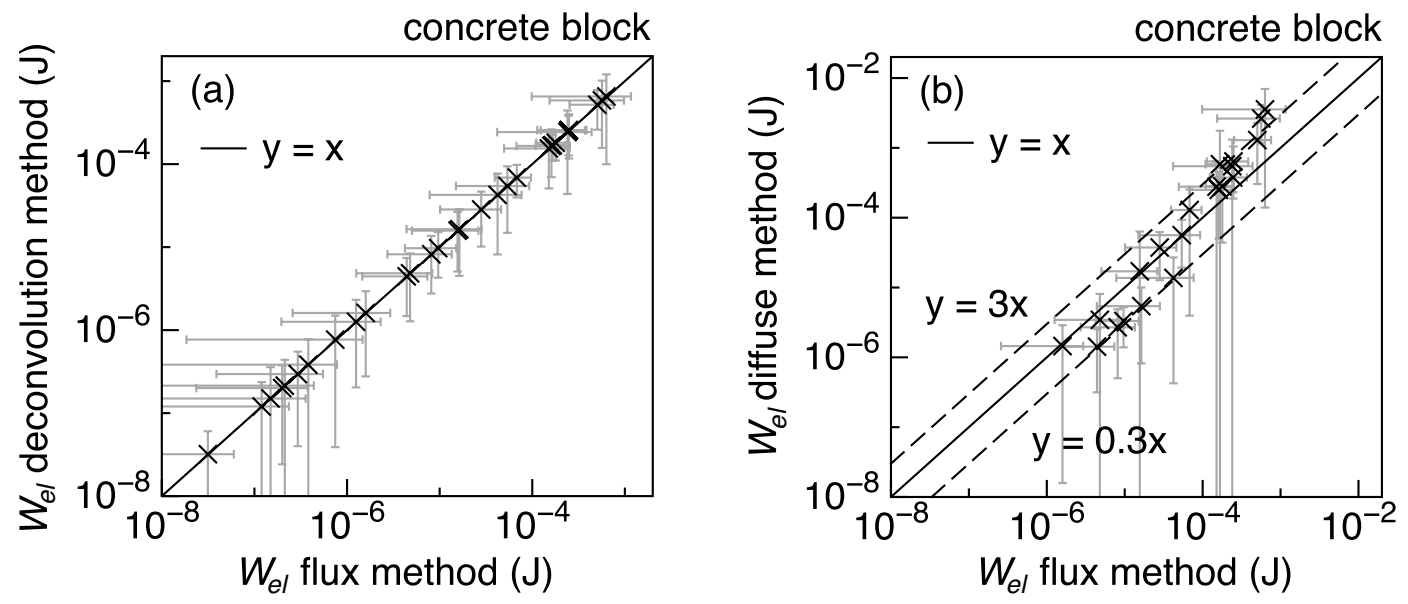

Figure 11: Comparison of the radiated elastic energy $W_{e l}$ calculated using the three methods [equations (31), (35) and (36)] for impacts of steel beads of various diameters from 2 $\mathrm{mm}$ to $20 \mathrm{~mm}$ dropped from various heights from $5 \mathrm{~cm}$ to $43 \mathrm{~cm}$ on the concrete block. Error bars ( \pm 1 standard deviation) are estimated from reproducibility tests conducted on a series of 12 identical experiments. 

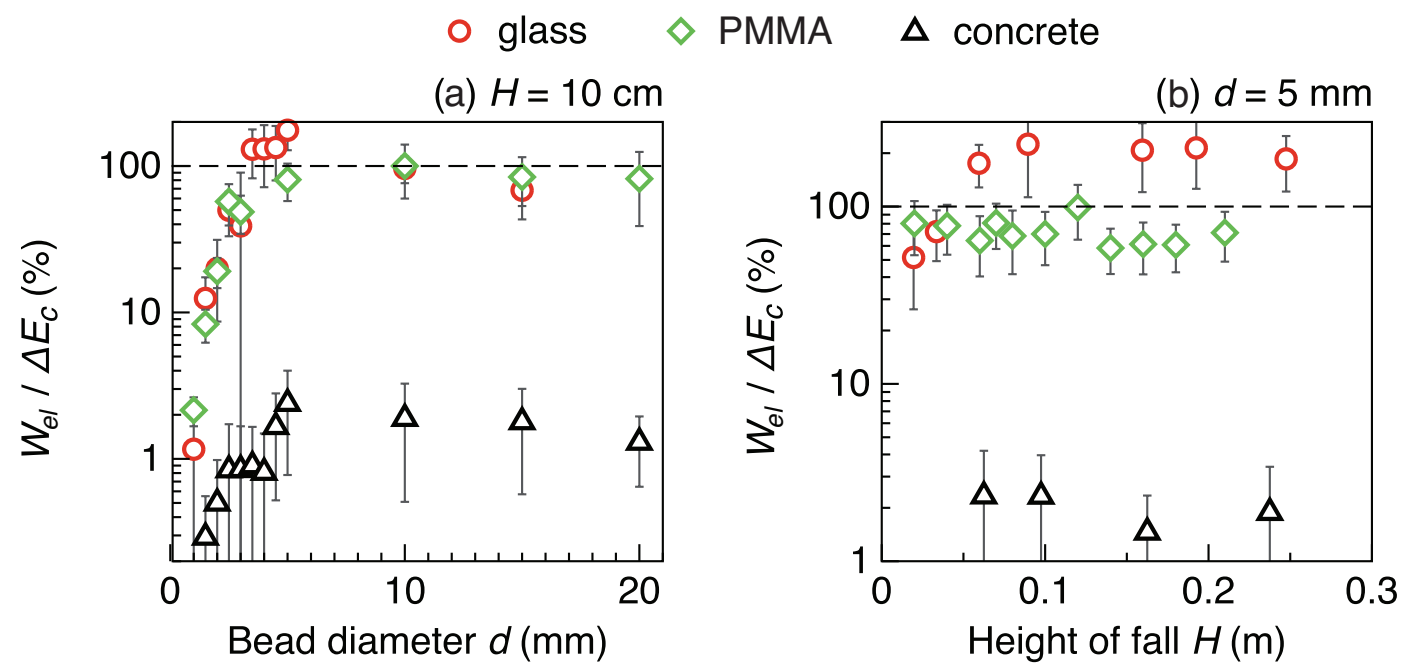

Figure 12: Ratio of the radiated elastic energy $W_{e l}$ to the energy lost during the impact $\Delta E_{c}$, as a function of (a) the bead diameter $d$ for drops tests from height $H=10 \mathrm{~cm}$ and (b) the fall height $H$ for a bead diameter $d=5 \mathrm{~mm}$, on the glass plate, PMMA plate and concrete block. Error bars ( \pm 1 standard deviation) are estimated from reproducibility tests conducted on a series of 12 identical experiments. 


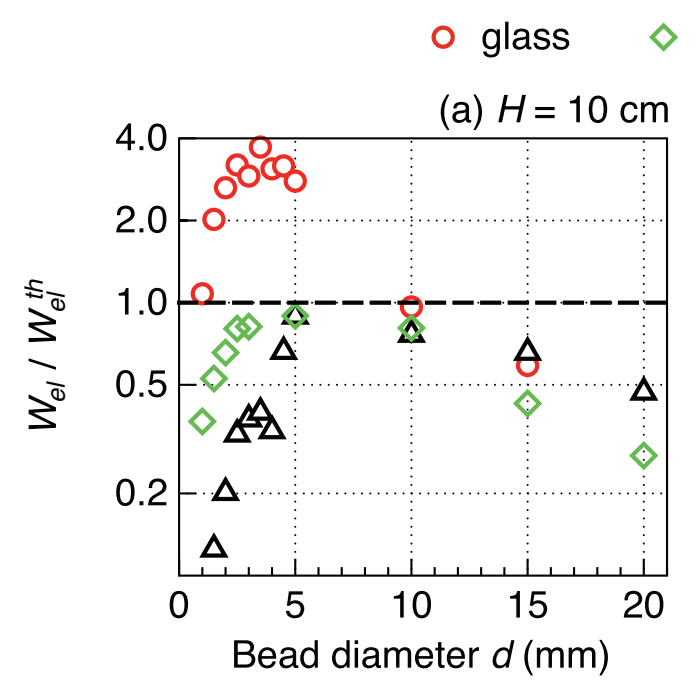

PMMA $\Delta$ concrete
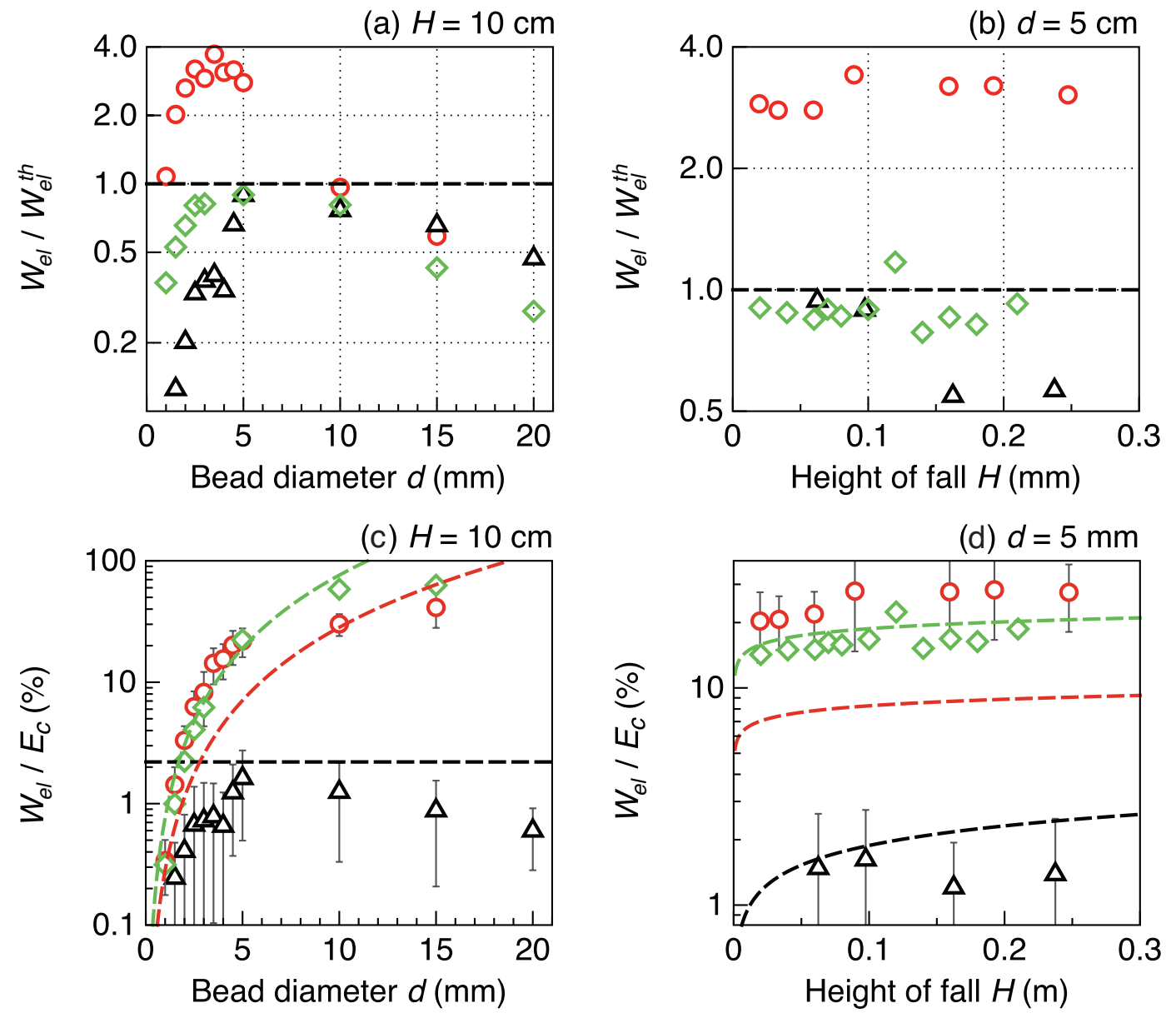

Figure 13: Ratio of the radiated elastic energy $W_{e l}$ measured with the energy flux method (a)-(b) to the theoretical radiated energy $W_{e l}^{t h}$ and (c)-(d) to the energy of the impact $E_{c}=\frac{1}{2} m V_{z}^{2}$, with $m$, the bead mass and $V_{z}$, the impact speed for impacts of steel beads of (a)-(c) different diameters $d$ for a fall height $H=10 \mathrm{~cm}$ and (b)-(d) different fall heights $H$ for a diameter $d=5 \mathrm{~cm}$, on the glass plate, PMMA plate and concrete block. In Figures (c) and (d), the dashed lines represent the ratio of the theoretical radiated elastic energy $W_{e l}^{t h}$ to $E_{c}$. 


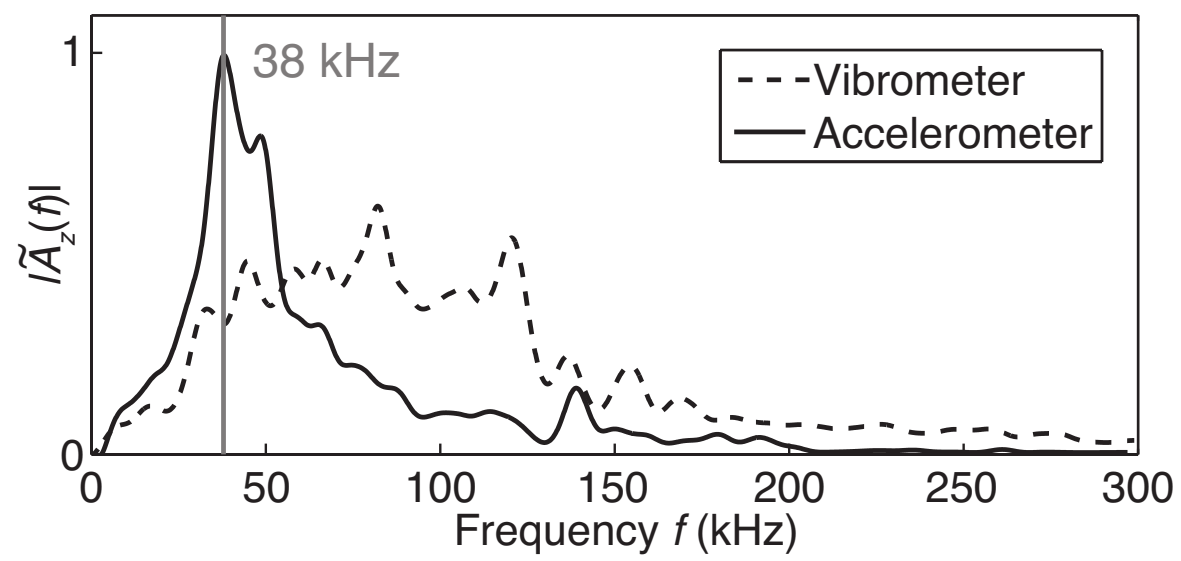

Figure 14: Normalized vibration acceleration $\left|\tilde{A}_{z}(f)\right|$ generated by the impact of a $3 \mathrm{~mm}$ steel bead on the surface of the glass plate. The vibration is recorded by a laser Doppler vibrometer (dashed line) and by the accelerometer used in this study (full line). The system constituted by the glass plate and the accelerometer shows a resonance around $38 \mathrm{kHz}$. Note that the accelerometer is not very sensitive to the frequencies higher than $100 \mathrm{kHz}$. However, most of the impacts investigated here generate signals with frequencies lower than $100 \mathrm{kHz}$. Practically, the laser Doppler vibrometer has a much lower signal to noise ratio than the accelerometer and therefore was not used in this study. 

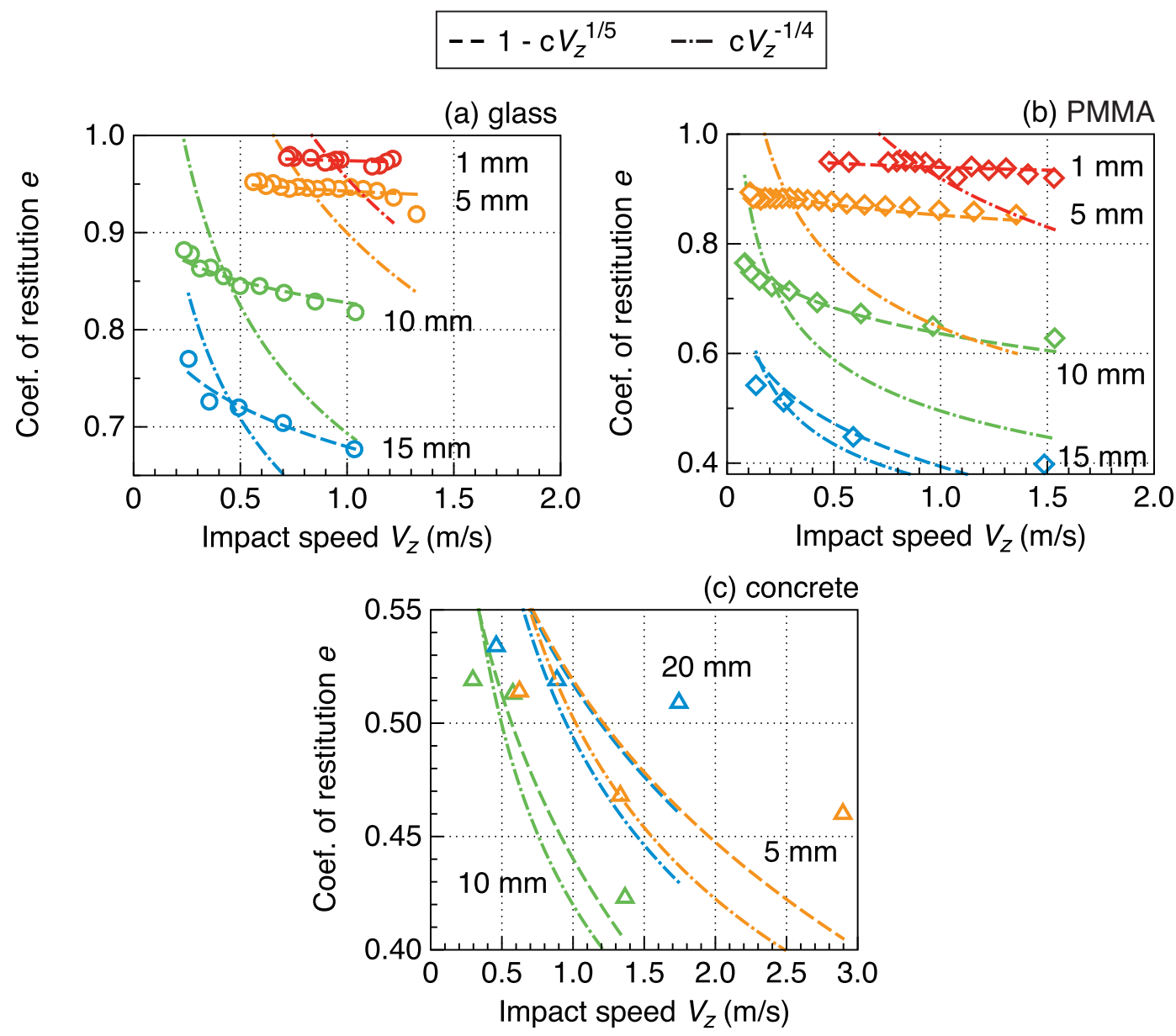

Figure 15: Coefficient of restitution $e$ as a function of the impact speed $V_{z}$ for different bead diameters $d$ (different colors) on the (a) glass plate, (b) PMMA plate and (c) concrete block. The dashed and dash-dotted lines represent the fitting of the experimental data with the scaling laws $e=1-c V_{z}^{1 / 5}$ and $e=c V_{z}^{-1 / 4}$, respectively, where $c$ is a constant that depends on the bead diameter. 

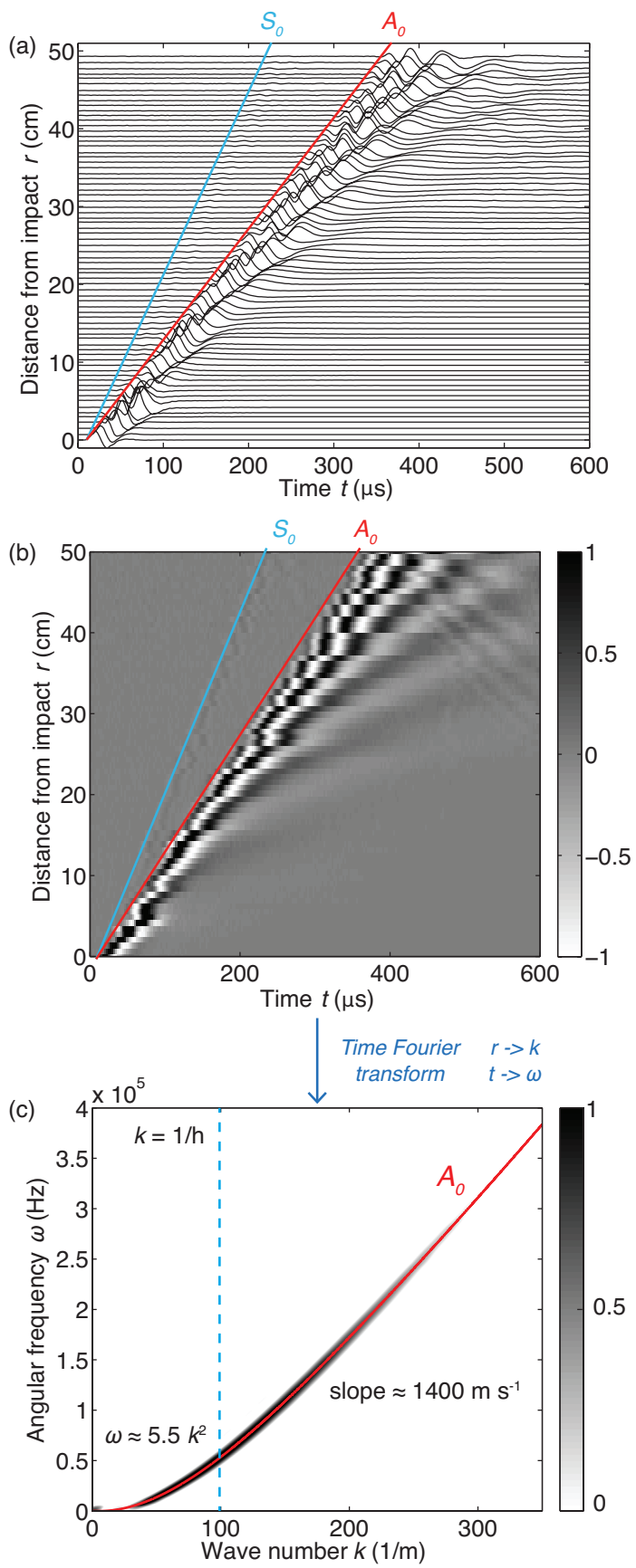

Figure 16: (a) Vibration acceleration $a_{z}(r, t)$ recorded at different distances $r$ from the impact of a 2-mm steel bead on the PMMA plate. The amplitude of each signal is normalized by its maximum value. The red and blue lines indicate the arrival of the Lamb modes $A_{0}$ and $S_{0}$, respectively. (b) Matrix representa 690 of the signals of (a). (c) Relation between the angular frequency $\omega$ and the wave number $k$ (i.e. dispersion relation), obtained by time and space Fourier transforms of the matrix in (b). Light and dark shading represent respectively low and high power spectral energy (normalized). Red line: theoretical dispersion relation for the fundamental mode of Lamb $A_{0}$ in a PMMA plate of thickness 

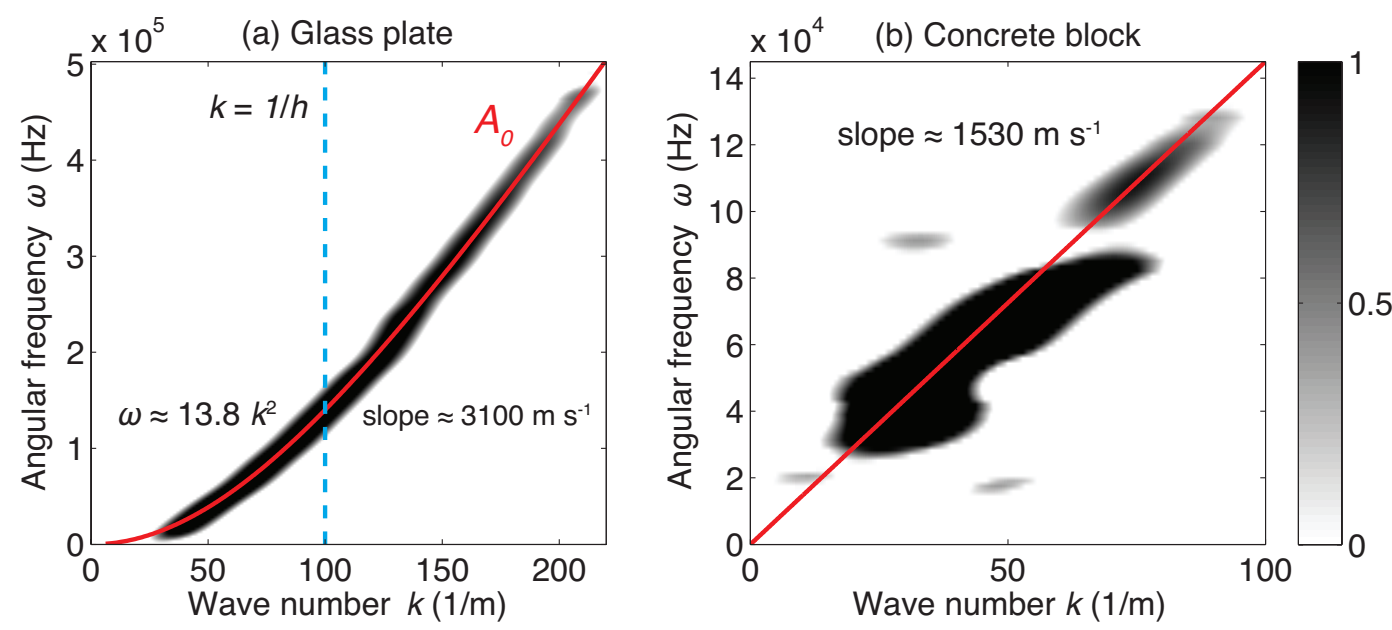

Figure 17: Relation between the angular frequency $\omega$ and the wave number $k$ (i.e. dispersion relation) for the direct wave front in (a) the glass plate and (b) the concrete block. Light and dark shading represent respectively low and high power spectral energy (normalized). Red line: (a) theoretical dispersion relation for the fundamental mode of Lamb $A_{0}$ in a glass plate of thickness $h=1 \mathrm{~cm}$ and elastic parameters reported in Table 2, (b) Linear fit of the data. In the concrete block, the group velocity $v_{g}=\partial \omega / \partial k$ equals the phase velocity $v_{\phi}=\omega / k$ and is about $1530 \mathrm{~m} \mathrm{~s}^{-1}$. 


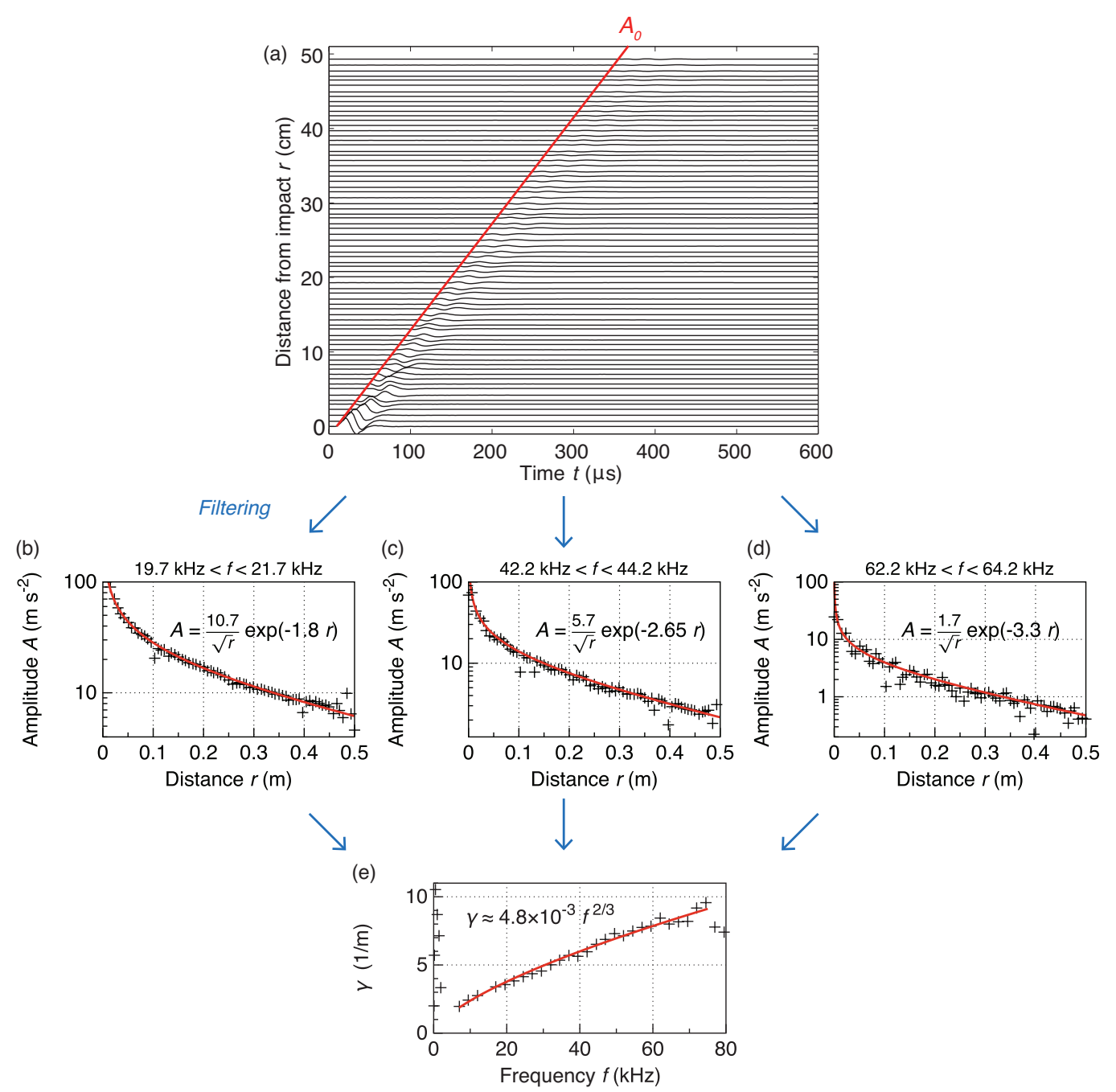

Figure 18: (a) Vibration acceleration $a_{z}(r, t)$ recorded at different distances $r$ from the impact of a 2-mm steel bead on the PMMA plate. (b), (c) and (d) The signals are filtered in different frequency ranges and their maximum amplitude is represented as a function of the distance $r$. (e) Attenuation coefficient $\gamma$ in the PMMA plate as a function of frequency $f$. 
(a)
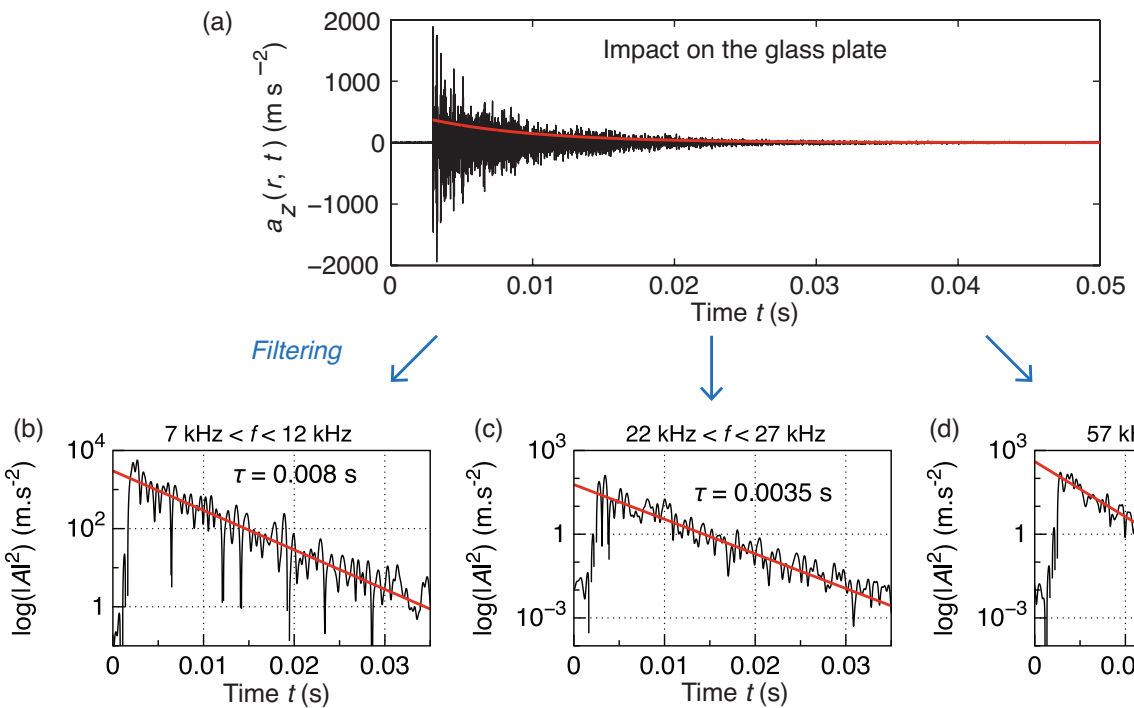

(c) $10^{3} \quad 22 \mathrm{kHz}<f<27 \mathrm{kHz}$
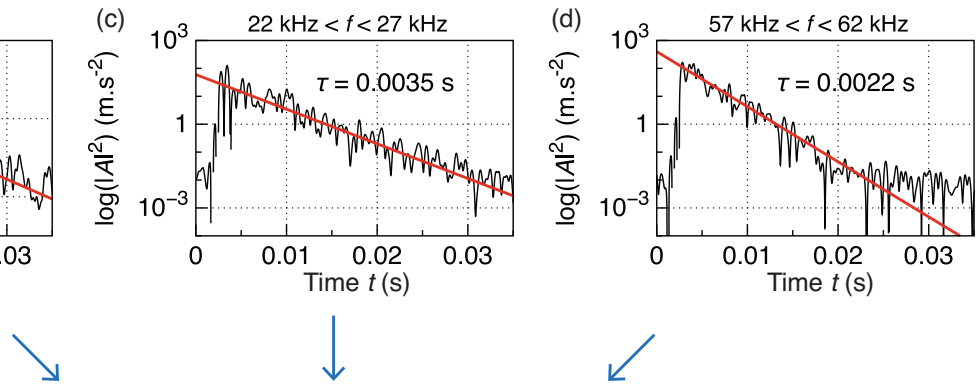

(e)

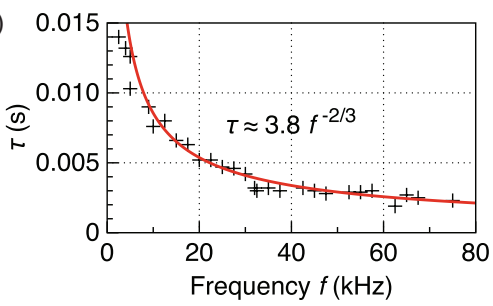

Figure 19: (a) Vibration acceleration $a_{z}(r, t)$ generated by the impact of a 4-mm steel bead on the glass plate. (b), (c) and (d) The vibration in (a) is filtered in different frequency ranges. The envelope of the squared vibration averaged over several periods decreases exponentially with time and the inverse of the slope in semi-logarithmic scale (red line) is the characteristic time $\tau$. (e) $\tau$ as a function of frequency $f$ in the glass plate. 

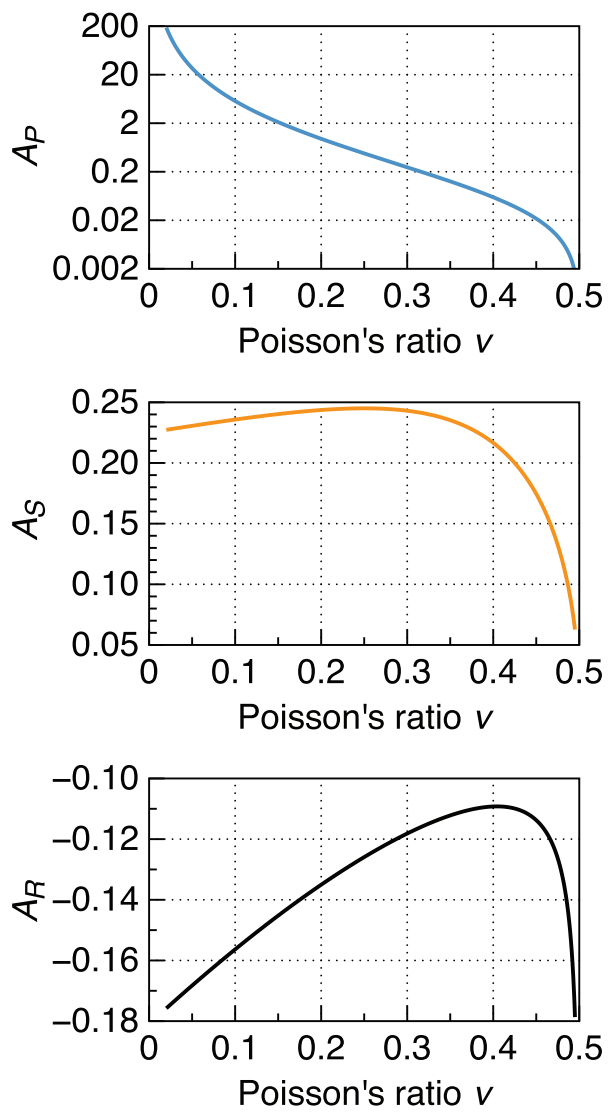

Figure 20: Values of the coefficient $A_{P}, A_{S}$ and $A_{R}$ as a function of Poisson's ratio $\nu$.

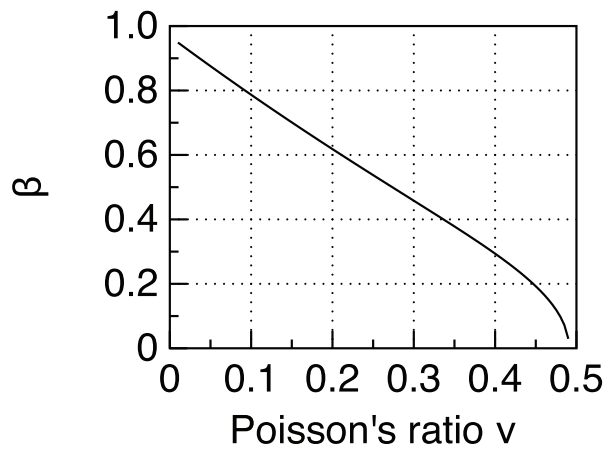

Figure 21: Coefficient $\beta$ defined by equation (33) as a function of the Poisson ratio $\nu$. 